\title{
MILES FORMULAE FOR BOOLEAN MODELS OBSERVED ON LATTICES
}

\author{
JOACHIM OHSER ${ }^{1}$, WERnER NAGEL ${ }^{2}$ AND KATJA SCHLADITZ ${ }^{3}$ \\ ${ }^{1}$ Hochschule Darmstadt, Fachbereich Mathematik und Naturwissenschaften, Schöfferstraße 3, D-64295 \\ Darmstadt, Germany; ${ }^{2}$ Friedrich-Schiller-Universität Jena, Institut für Stochastik, D-07737 Jena, Germany; \\ ${ }^{3}$ Fraunhofer-Institut für Techno- und Wirtschaftsmathematik, Abteilung Bildverarbeitung, Fraunhofer-Platz 1, \\ D-67663 Kaiserslautern, Germany \\ e-mail: jo@h-da.de,nagel@minet.uni-jena.de,katja.schladitz@itwm.fraunhofer.de
}

(Accepted May 12, 2009)

\begin{abstract}
The densities of the intrinsic volumes - in 3D the volume density, surface density, the density of the integral of the mean curvature and the density of the Euler number - are a very useful collection of geometric characteristics of random sets. Combining integral and digital geometry we develop a method for efficient and simultaneous calculation of the intrinsic volumes of random sets observed in binary images in arbitrary dimensions. We consider isotropic and reflection invariant Boolean models sampled on homogeneous lattices and compute the expectations of the estimators of the intrinsic volumes. It turns out that the estimator for the surface density is proved to be asymptotically unbiased and thus multigrid convergent for Boolean models with convex grains. The asymptotic bias of the estimators for the densities of the integral of the mean curvature and of the Euler number is assessed for Boolean models of balls of random diameters. Miles formulae with corresponding correction terms are derived for the 3D case.
\end{abstract}

Keywords: Boolean models, digital topology, image analysis, Miles' formulae, Minkowski functionals, quermass densities, volume images.

\section{INTRODUCTION}

With the fast development of new materials like foams or fibre reinforced composites there is a growing need for non-destructive testing and structure characterisation. Often, geometric characteristics of the whole structure have to be estimated from the given $2 \mathrm{D}$ or $3 \mathrm{D}$ image of a small sample. A very attractive set of geometric characteristics are the intrinsic volumes (or quermassintegrals or Minkowski functionals). On the one hand, they include the widely used volume $V$, surface area $S$, and Euler number $\chi$. On the other hand, they are in some sense complete, see Hadwiger (1957). The fourth intrinsic volume in $3 \mathrm{D}$ - the integral of mean curvature $M$ - has nice interpretations for various structures, too. In particular for random fibre systems, it is proportional to the total fibre length.

Throughout this paper, we assume that the structure of interest is observed in an $n$-dimensional binary digital image. That is, the structure is given by its intersection with a lattice and a cuboidal window and values associated the lattice points indicating structure (1) or background (0).

The well known integral geometric Crofton formulae provides a method for determining the intrinsic volumes from lower dimensional sections of the structure. A discretisation of this formula is the basis of our method, see also Chapter 4 in Ohser and Mücklich (2000) and Lang et al. (2001). The Crofton formulae reduce the estimation of intrinsic volumes to the estimation of the Euler number in lower dimensional sections. Therefore, the present paper is based on thorough investigations of digital connectivity and consistent estimation of the Euler number, see Nagel et al. (2000); Ohser et al. (2002; 2003); Schladitz et al. (2006). The consistency results for the Euler number estimates of foreground and background established in Ohser et al. (2002) carry over to all intrinsic volumes. In order to give the reader a comprehensive description of our approach making the latter as self-contained as possible, we recall some facts addressed in the papers mentioned above. In the present paper it is shown that this approach can be extended on all intrinsic volumes in arbitrary dimensions.

In order to check the accuracy of the proposed method, the bias of the estimators is computed for macroscopically homogeneous (stationary) and isotropic Boolean models. Using ideas of Serra (1982), we show that our surface area estimator is multigrid convergent for this very rich class of sets. However, the bias of the estimators for the integral of mean curvature and for the Euler number does not necessarily vanish with decreasing lattice distance. Even worse, for three out of the four adjacency systems examined, the bias 
of the Euler number estimator diverges. Nevertheless, for lattice distances in the range of $1 \%$ to $10 \%$ of the mean grain size of the Boolean model, the estimation is sufficiently accurate for practical applications. The explicit formulae for the bias of the estimators lead to formulae connecting the estimated intrinsic volumes with the parameters of the Boolean model. The structure of these formulae resembles the original Miles' formulae (Miles, 1976) in the Euclidean space with additional terms correcting for the discretisation effects.

The presented method yields a fast algorithm for simultaneously determining the intrinsic volumes and their densities. The core of the algorithm consists in a convolution of the binary image with a suitably chosen $2^{n}$ mask resulting in a grey value image, see Lang et al. (2001); Ohser and Mücklich (2000). All further steps are based solely on the grey value histogram whose size does not depend on image size or content. Thus the advantage over other methods for estimating the intrinsic volumes, see Blasquez and Poiraudeau (2003); Schmidt and Spodarev (2005) are simplicity and speed of the algorithm. The surface area in 3D is measured directly from the binary volume image without need to approximate the surface. Moreover, compared to empirical marching cubes methods like in Windreich et al. (2003), only a small number of pixel configurations has to be taken into account. In Schladitz et al. (2006) the weights for the surface are compared with those of Lindblad and Nyström (2002), see also Ziegel and Kiderlen (2009) for a sound investigation of surface area estimation.

This paper is organised as follows: First we repeat some facts about homogeneous lattices and summarise results on adjacency systems and Euler number estimation from Nagel et al. (2000); Ohser et al. (2002; 2003); Schladitz et al. (2006). Based on concepts of section lattices and their translative complements, we derive discrete versions of the Crofton formulae for polyconvex sets and from these, estimators for the densities of the intrinsic volumes of random sets. In particular, we compute weights for the estimation of the density of the integral of the mean curvature in $\mathbb{R}^{3}$. Finally, we present Miles' formulae for Boolean models in $\mathbb{R}^{n}$ sampled on homogeneous lattices. In principle, we follow Ohser et al. (2003) where formulae are given for the density of the Euler number of Boolean models in $\mathbb{R}^{3}$ with balls of constant diameter. These results are generalised as follows: We derive formulae for the expectations of the densities of the intrinsic volumes for Boolean models with convex grains. Advantages and drawbacks of the presented method are discussed in the last section.

\section{POINT LATTICES AND PIXEL CONFIGURATIONS}

Image data are usually given on homogeneous point lattices, e.g., the cubic primitive lattice $\mathbb{L}^{n}=a \mathbb{Z}^{n}$, $a>0$, where $\mathbb{Z}$ denotes the set of integers and $a$ is the lattice distance. Of course, most images are given on orthogonal lattices. However, we will consider lower dimensional section lattices of cubic or orthorhombic lattices, too, which are not necessarily orthogonal. Hence we introduce homogeneous lattices in a more general setting.

Let $u_{1}, \ldots, u_{n} \in \mathbb{R}^{n}$ form a basis of $\mathbb{R}^{n}$ and let $U=\left(u_{1}, \ldots, u_{n}\right)$ be the matrix of column vectors. Then $\mathbb{L}^{n}=U \mathbb{Z}^{n}$ is called an $n$-dimensional homogeneous lattice. The closed unit cell of $\mathbb{L}^{n}$ w.r.t. the basis $u_{1}, \ldots, u_{n}$ is the Minkowski sum $C=\left[0, u_{1}\right] \oplus \ldots \oplus$ $\left[0, u_{n}\right]$ of the segments $\left[0, u_{i}\right]$ between the origin 0 and the lattice points $u_{i}$. Its volume is $\operatorname{vol} C=|\operatorname{det} U|>0$, and the value of $|\operatorname{det} U|$ does not depend on the choice of the lattice basis.

The intersection $X \cap \mathbb{L}^{n}$ of a compact set $X \subset \mathbb{R}^{n}$ and a homogeneous lattice $\mathbb{L}^{n}$ is called a $\mathbb{L}^{n}$-sampling of $X$. In the language of image processing, $X \cap \mathbb{L}^{n}$ is the set of foreground pixels and $X^{c} \cap \mathbb{L}^{n}$ is the background.

Locally, an $\mathbb{L}^{n}$-sampling of $X$ can be described by pixel configurations which are specified as follows: The vertices of the unit cell $C$ are indexed, and we write $x_{j}=\sum_{i=1}^{n} \lambda_{i} u_{i}$ with the index $j=\sum_{i=1}^{n} 2^{i-1} \lambda_{i}$, $\lambda_{i} \in\{0,1\}$. Clearly, the unit cell $C$ has $2^{n}$ vertices, $x_{j} \in \mathscr{F}^{0}(C), j=0, \ldots, 2^{n}-1$, where $\mathscr{F}^{0}(C)$ denotes the set of vertices of $C$. In a similar way we introduce the indices for all subsets $\xi \subseteq \mathscr{F}^{0}(C)$. Let $\mathbf{1}$ denote the indicator function, i.e., $\mathbf{1}(x \in \xi)=1$ if $x \in \xi$ and $\mathbf{1}(x \in \xi)=0$ otherwise. An index $\ell$ is assigned to a $\xi$, and we write $\xi_{\ell}$ if

$$
\ell=\sum_{j=0}^{2^{n}-1} 2^{j} \cdot \mathbf{1}\left(x_{j} \in \xi\right),
$$

for all $\xi \subseteq \mathscr{F}^{0}(C)$. We have $\ell \in\{0, \ldots, v\}$ with $v=$ $2^{2^{n}}-1$. That means, the powers of 2 are used for encoding the vertices. Notice that $\xi_{0}=\emptyset, \xi_{v}=\mathscr{F}^{0}(C)$, and $\xi_{v-\ell}=\xi_{v} \backslash \xi_{\ell}$. The set $\xi_{\ell}$ can be considered as a local pixel configuration of the foreground.

We use pictograms to illustrate configurations where full discs mark foreground pixels and empty discs mark background pixels, see Table 1 for examples. Sets of pixel configurations are denoted by pictograms with vertices not marked with a disc, which can be either foreground or background pixels, see Table 2.

In the case of a cubic primitive lattice which is highly symmetric, the pixel configurations $\xi_{\ell} \subseteq \mathscr{F}^{0}(C)$ 
are commonly grouped in equivalence classes. Let $\mathscr{M}$ be the set of all linear isometries (rigid motions and reflections) leaving $\mathbb{L}^{n}$ unchanged, i.e., $\theta \mathbb{L}^{n}=\mathbb{L}^{n}$ for all $\theta \in \mathscr{M}$. The configuration $\xi_{\ell_{1}}$ is equivalent to $\xi_{\ell_{2}}$ if there is a $\theta \in \mathscr{M}$ and a $y \in \mathbb{L}^{n}$ such that $\theta \xi_{\ell_{1}}+y=$ $\xi_{\ell_{2}}$. By $D_{0}, \ldots, D_{v_{0}}$ we denote the congruence classes of $\left\{\xi_{0}, \ldots, \xi_{v}\right\}$ under $\mathscr{M}$. Furthermore, we choose a system $\left\{\eta_{0}, \ldots, \eta_{v_{0}}\right\}$ of representatives $\eta_{j} \in D_{j}$. We write $\eta_{j}^{c}$ for a representative of the equivalence class of $\mathscr{F}^{0}(C) \backslash \eta_{j}$.

Since the intrinsic volumes are motion invariant characteristics, their weights can be presented in terms of the congruence classes instead for the complete set of pixel configurations. The $v_{0}+1=22$ congruence classes in the $3 \mathrm{D}$ case are given in Table 1 with our choice of representatives $\eta_{0}, \ldots, \eta_{21}$.

\section{ADJACENCY AND EULER NUMBER}

In the literature the adjacency relation of lattice points (or pixels) is usually characterised by a neighbourhood graph and the complementarity of adjacencies is defined via the Jordan surface theorem (Jordan-Brouwer theorem), see, e.g., Lachaud and Montanvert (2000). Here we follow Ohser et al. (2003); Schladitz et al. (2006) where the definition of adjacency is based on a consistency relation for the Euler number.

We first consider the convex hulls $F_{\ell}=\operatorname{conv} \xi_{\ell}$ of a configuration $\xi_{\ell}$ forming convex polytopes with $F_{\ell} \subseteq C$ and $\mathscr{F}^{0}\left(F_{\ell}\right) \subseteq \mathscr{F}^{0}(C), \ell=1, \ldots, v$. We set $F_{0}=\emptyset$. Let $\mathscr{F}^{j}(F)$ denote the set of all $j$-dimensional faces of a convex polytope $F$. For a set $\mathbb{F}$ of convex polytopes write $\mathscr{F}^{j}(\mathbb{F})=\bigcup\left\{\mathscr{F}^{j}(F): F \in \mathbb{F}\right\}$ using the convention $\bigcup\left\{\mathscr{F}^{j}(F): F \in \mathbb{F}\right\}=\bigcup_{F \in \mathbb{F}} \mathscr{F}^{j}(F)$. Furthermore, by $\bar{A}$ we denote the topological closure of a set $A \subset \mathbb{R}^{n}$ and $\# N$ denotes the number of elements of a finite set $N$.

Definition 1 Let $\mathbb{F}_{\text {loc }} \subseteq\left\{F_{0}, \ldots, F_{v}\right\}$ be a set of convex polytopes $F_{\ell}=\operatorname{conv} \xi_{\ell}$ fulfilling the conditions

(i) $\emptyset \in \mathbb{F}_{\text {loc }}, C \in \mathbb{F}_{\text {loc }}$,

(ii) if $F \in \mathbb{F}_{\text {loc }}$ then $\mathscr{F}^{i}(F) \subset \mathbb{F}_{\text {loc }}$ for $i=0, \ldots, \operatorname{dim} F$,

(iii) if $F_{i}, F_{j} \in \mathbb{F}_{\text {loc }}$ and $\operatorname{conv}\left(F_{i} \cup F_{j}\right) \notin \mathbb{F}_{\text {loc }}$ then $F_{i} \cap F_{j}$, $\overline{F_{i} \backslash F_{j}}, \overline{F_{j} \backslash F_{i}} \in \mathbb{F}_{\text {loc }}$.

(iv) if $F_{i_{1}}, \ldots, F_{i_{m}} \in \mathbb{F}_{\text {loc }}$ and $F=\bigcup_{j=1}^{m} F_{i_{j}}$ is convex then $F \in \mathbb{F}_{\text {loc }}, m=2, \ldots, \# \mathbb{F}_{\text {loc }}$.
Then $\mathbb{F}_{\text {loc }}$ is called a local adjacency system and $\mathbb{F}=$ $\bigcup_{x \in \mathbb{L}^{n}}\left(\mathbb{F}_{\text {loc }}+x\right)$ is said to be an adjacency system of the lattice $\mathbb{L}^{n}$.

From (i) it immediately follows that $\mathscr{F}^{0}(\mathbb{F})=\mathbb{L}^{n}$. The conditions (ii) and (iii) ensure that the Euler number of the unions $\bigcup\left\{F \in \mathbb{F}: \mathscr{F}^{0}(F) \subset \xi_{\ell}\right\}$, $\ell=0, \ldots, v$, can be computed via the Euler-Poincaré formula and the inclusion-exclusion principle, see Schneider (1993). Condition (iv) prevents the existence of many different local adjacency systems generated from the same 'basic bricks'.

The pair $\Gamma=\left(\mathscr{F}^{0}(\mathbb{F}), \mathscr{F}^{1}(\mathbb{F})\right)$ is said to be the neighbourhood graph of $\mathbb{F}$; it consists of the set $\mathscr{F}^{0}(\mathbb{F})$ of vertices and the set $\mathscr{F}^{1}(\mathbb{F})$ of edges. All vertices are of the same order since $\Gamma$ is homogeneous, $\Gamma+$ $x=\Gamma, x \in \mathbb{L}^{k}$. The order of the vertices is called the connectivity of $\mathbb{L}^{n}$. We write $\mathbb{F}_{m}$ if the corresponding neighbourhood graph is $m$-connected.

For $n=3$ we recall the adjacency systems $\mathbb{F}_{6}$, $\mathbb{F}_{14.1}, \mathbb{F}_{14.2}$, and $\mathbb{F}_{26}$ considered in detail in Ohser et al. (2002; 2003).

- $\mathbb{F}_{6}$ is generated by the singleton containing the unit cell $C$, i.e., $\mathbb{F}_{6}=\bigcup_{x \in \mathbb{L}^{3}} \bigcup_{j=0}^{3} \mathscr{F}^{j}(C+x)$.

- $\mathbb{F}_{14.1}$ is generated by the tessellation of $C$ into the 6 tetrahedra $F_{139}, F_{141}, F_{163}, F_{177}, F_{197}, F_{209}$, i.e., $\mathbb{F}_{14.1}$ is the smallest adjacency system containing these tetrahedra.

- $\mathbb{F}_{14.2}$ is generated by the family of tetrahedra $F_{43}$, $F_{141}, F_{149}, F_{169}, F_{177}$, and $F_{212}$.

- The maximum adjacency system $\mathbb{F}_{26}$ on $\mathbb{L}^{3}$ is generated by $\mathbb{F}_{\text {loc }}=\left\{F_{0}, \ldots, F_{255}\right\}$.

Now we introduce a discretisation of a set w. r.t. a given adjacency system.

Definition 2 The discretisation $X \sqcap \mathbb{F}$ of a compact subset $X \subset \mathbb{R}^{n}$ w.r.t. a given adjacency system $\mathbb{F}$ is defined as the union of all elements of $\mathbb{F}$ that have all their vertices in $X$, i.e.,

$$
X \sqcap \mathbb{F}=\bigcup\left\{F \in \mathbb{F}: \mathscr{F}^{0}(F) \subseteq X\right\} .
$$

An adjacency system $\mathbb{F}$ can be seen as a set of 'bricks' of the discretisation where a 'brick' $F \in \mathbb{F}$ is a subset of the discretisation of $X$ if and only if all vertices of $F$ belong to $X$. Notice that $X \sqcap \mathbb{F}=\left(X \cap \mathbb{L}^{n}\right) \sqcap \mathbb{F}$, i.e., the discretisation $X \sqcap \mathbb{F}$ is determined by the $\mathbb{L}^{n}$-sampling of $X$ alone.

As in Ohser et al. (2002; 2003); Schladitz et al. (2006) we approximate $X$ by a discretisation $X \sqcap \mathbb{F}$ w. r. t. an adjacency system $\mathbb{F}$. Since the set $X \sqcap \mathbb{F}$ forms a (not necessarily convex) polyhedron, the number $\#_{\mathscr{F}^{j}}(X \sqcap \mathbb{F})$ of elements of $\mathscr{F}^{j}(X \sqcap \mathbb{F})$ is finite and, 
therefore, the Euler number $\chi(X \sqcap \mathbb{F})$ can be computed via the Euler-Poincaré formula,

$$
\chi(X \sqcap \mathbb{F})=\sum_{j=0}^{n}(-1)^{j} \# \mathscr{F}^{j}(X \sqcap \mathbb{F}) .
$$

In order to apply a "local method" for measuring the Euler number we consider a local version of the Euler-Poincaré formula (Eq. 3). The conditions (ii) and (iii) in Definition 1 ensure that the Euler number of a discretisation $X \sqcap \mathbb{F}$ can be computed from local knowledge. We observe that for the local configuration $\xi=X \cap C \cap \mathbb{L}^{n}$ of $X \cap \mathbb{L}^{n}$ we have $\xi \sqcap \mathbb{F}=(X \sqcap \mathbb{F}) \cap C$. Now we define the weights

$$
\mu_{F}=\min \left\{j: \text { there is a } G \in \mathscr{F}^{j}(C) \text { with } F \subseteq G\right\},
$$

for correcting effects occurring at the cells' edges. The expression $2^{n-\mu_{F}}$ is the number of lattice cells covering $F$, i.e., the face $F$ belongs to totally $2^{n-\mu_{F}}$ neighbouring cells. Thus, the reciprocal weight $2^{\mu_{F}-n}$ should be used when counting $F$ locally. Now the number $\chi_{0}^{n}$ can be introduced as

$$
\chi_{0}^{n}\left(\xi_{\ell} \sqcap \mathbb{F}\right)=\sum_{j=0}^{n}(-1)^{j} \sum_{F \in \mathscr{F} j\left(\xi_{\ell} \sqcap \mathbb{F}\right)} 2^{\mu_{F}-n},
$$

for $\ell=0, \ldots, v$. We have $\chi_{0}^{n}\left(\left(\xi_{\ell}+x\right) \sqcap \mathbb{F}\right)=\chi_{0}^{n}\left(\xi_{\ell} \sqcap \mathbb{F}\right)$ for all $x \in \mathbb{L}^{n}$, cf. also Jernot et al. (2004). In fact, $\chi_{0}^{n}$ can be considered as an edge-corrected localisation of $\chi$. One observes that

$$
\sum_{F \in \mathscr{F} j\left(\xi_{\ell} \sqcap \mathbb{F}\right)} 2^{\mu_{F}-n} \sum_{x \in \mathbb{L}^{n}} \mathbf{1}(F+x \subseteq C)=\# \mathscr{F} j\left(\xi_{\ell} \sqcap \mathbb{F}\right),
$$

for all $\xi_{\ell} \subseteq \mathscr{F}^{0}(C)$. Now, the additivity and translation invariance of the Euler number and the fact that $X \sqcap$ $\mathbb{F}=\left(X \cap \mathbb{L}^{n}\right) \sqcap \mathbb{F}$ yield

$$
\begin{aligned}
& \chi(X \sqcap \mathbb{F})=\sum_{x \in \mathbb{L}^{n}} \chi_{0}^{n}(C \cap((X \sqcap \mathbb{F})-x)) \\
& =\sum_{x \in \mathbb{L}^{n}} \sum_{\ell=0}^{v} \chi_{0}^{n}\left(\xi_{\ell} \sqcap \mathbb{F}\right) \mathbf{1}\left(\xi_{\ell}+x \subseteq X\right) \mathbf{1}\left(\xi_{v-\ell}+x \subseteq X^{c}\right) \\
& =\sum_{\ell=0}^{v} \underbrace{\chi_{0}^{n}\left(\xi_{\ell} \sqcap \mathbb{F}\right)}_{w_{\ell}} \underbrace{\sum_{x \in \mathbb{L}^{n}} \mathbf{1}\left(\xi_{\ell}+x \subseteq X\right) \mathbf{1}\left(\xi_{v-\ell}+x \subseteq X^{c}\right)}_{h_{\ell}}
\end{aligned}
$$

with the convention $0 \cdot \infty=0$. The last equation shows that the Euler number can be written as a scalar product of the vectors $w=\left(w_{\ell}\right)$ and $h=\left(h_{\ell}\right)$. The components $w_{\ell}=\chi_{0}^{n}\left(\xi_{\ell} \sqcap \mathbb{F}\right)$ of the vector $w=\left(w_{\ell}\right)$ serve as weights for computing the Euler number. It is important to realise that these weights depend on $\mathbb{F}$, but they are independent of $X$. On the other hand, the vector $h=\left(h_{\ell}\right)$ is independent of $\mathbb{F}$. The components of $w$ for the adjacency systems $\mathbb{F}_{6}, \mathbb{F}_{14.1}, \mathbb{F}_{14.2}$ and $\mathbb{F}_{26}$ on $\mathbb{L}^{3}$ are given in Ohser et al. (2003).

Now following Jernot et al. (2004), we consider a refinement of Eq. 3 which is possible when $\mathbb{F}$ is generated by a tessellation $\mathscr{G}_{0}=\left\{P_{1}, \ldots, P_{m}\right\} \subseteq$ $\left\{F_{0}, \ldots, F_{v}\right\}$ of the unit cell $C$. The set $P_{i}$ are $n$ dimensional polytopes with $\bigcup_{i=1}^{m} P_{i}=C$ and $\operatorname{int} P_{i} \cap$ $\operatorname{int} P_{j}=\emptyset$ for $i \neq j$, where int $P$ denotes the interior of $P$. Then the periodic extension $\mathscr{G}=\bigcup_{x \in \mathbb{L}^{n}}\left(\mathscr{G}_{0}+x\right)$ is a (deterministic) tessellation of $\mathbb{R}^{n}$. We assume that $\mathscr{G}$ forms a face-to-face tessellation. As in Ohser et al. (2002), we call a tessellation $\mathscr{G}$ having the above properties an admissible tessellation.

Let $\tilde{\mu}_{F}$ be the number of cells of $\mathscr{G}$ covering $F$,

$$
\tilde{\mu}_{F}=\#\{P \in \mathscr{G}: F \subseteq P\},
$$

for $F \in \mathscr{F}^{j}\left(P_{i}\right)$ and $j=1, \ldots, n$. We follow Jernot et al. (2004) where an alternative version $\tilde{\chi}_{0}^{n}$ of the local Euler number is defined as

$$
\begin{aligned}
& \tilde{\chi}_{0}^{n}\left(\xi_{\ell} \sqcap \mathbb{F}\right)= \\
& \sum_{i=1}^{m} \underbrace{\sum_{j=0}^{n}(-1)^{j} \sum_{F \in \mathscr{F}^{j}\left(P_{i}\right)} \frac{\mathbf{1}\left(\mathscr{F}^{0}(F) \subseteq \xi_{\ell} \cap P_{i}\right)}{\tilde{\mu}_{F}}}_{\tilde{w}_{\ell}^{(i)}} .
\end{aligned}
$$

It follows that $\tilde{\chi}_{0}^{n}=\chi_{0}^{n}$ and thus Eq. 6 is valid with $\tilde{\chi}_{0}^{n}$ instead of $\chi_{0}^{n}$.

Finally, we introduce pairs of complementary adjacency systems. In the continuous case the consistency relation $\chi(X)=(-1)^{n+1} \chi\left(\overline{X^{c}}\right)$ is fulfilled for all compact, polyconvex and topologically regular sets $X \subset \mathbb{R}^{n}$, see Ohser et al. (2002); Rataj and Zähle (2003), and a similar relationship should hold in the discrete case.

Definition 3 The pair $\left(\mathbb{F}, \mathbb{F}_{c}\right)$ is called a pair of complementary adjacency systems if $(X \sqcap \mathbb{F}) \cap\left(X^{c} \sqcap\right.$ $\left.\mathbb{F}_{c}\right)=\emptyset$ and

$$
\chi(X \sqcap \mathbb{F})=(-1)^{n+1} \chi\left(X^{c} \sqcap \mathbb{F}_{c}\right),
$$

for all compact $X \subset \mathbb{R}^{n}$. An adjacency system $\mathbb{F}$ is called self-complementary if $(X \sqcap \mathbb{F}) \cap\left(X^{c} \sqcap \mathbb{F}\right)=\emptyset$ and $\chi(X \sqcap \mathbb{F})=(-1)^{n+1} \chi\left(X^{c} \sqcap \mathbb{F}\right)$ for all compact $X$.

For a given adjacency system $\mathbb{F}$ there does not necessarily exist a complementary adjacency system $\mathbb{F}_{c}$, and until now there is not known a constructive way to find $\mathbb{F}_{c}$. Furthermore, the criterion of Definition 
3 is not appropriate for checking complementarity. A simpler criterion is given in Schladitz et al. (2006).

It turns out that the 6-adjacency on $\mathbb{L}^{3}$ is complementary to the 26-adjacency and there are two self-complementary adjacency systems known on $\mathbb{L}^{3}$, the 14.1-adjacency and the 14.2-adjacency. In other words, $\left(\mathbb{F}_{6}, \mathbb{F}_{26}\right),\left(\mathbb{F}_{14.1}, \mathbb{F}_{14.1}\right),\left(\mathbb{F}_{14.2}, \mathbb{F}_{14.2}\right)$ and $\left(\mathbb{F}_{26}, \mathbb{F}_{6}\right)$, are pairs of complementary adjacency systems on $\mathbb{L}^{3}$, see also Ohser et al. (2002; 2003); Schladitz et al. (2006).

\section{INTRINSIC VOLUMES}

The Crofton formulae reduce measurement of the intrinsic volumes to computation of Euler numbers in lower dimensional sections. A discretisation of these formulae combined with an efficient calculation of the Euler numbers in the intersections yield a fast algorithm for simultaneously determining the intrinsic volumes from an $\mathbb{L}^{n}$-sampling of $X$. The backbone of the Euler number calculation is a thorough investigation of digital connectivity and consistency.

\section{SECTION LATTICES AND TRANSLATION LATTICES}

Let $X \subset \mathbb{R}^{n}$ be a compact set (i.e., an object or a particle). We assume that $X$ is polyconvex, i.e., $X$ is a finite union of compact convex sets. The Crofton formulae for computing the intrinsic volumes of $X$ use section profiles of $X$ on affine subspaces of $\mathbb{R}^{n}$. In order to obtain a digitised version, we introduce section lattices of a given homogeneous lattice $\mathbb{L}^{n}$ and their translative complements in analogy to linear subspaces and their orthogonal complements.

Definition 4 A pair $\left(\mathbb{L}^{k},{ }^{T} \mathbb{L}^{n-k}\right), k=1, \ldots, n-1$, is called a $k$-dimensional section lattice $\mathbb{L}^{k}$ equipped with the translative complement ${ }^{T} \mathbb{L}^{n-k}$, if there exists a basis $\mathrm{v}_{1}, \ldots, \mathrm{v}_{n}$ of $\mathbb{L}^{n}$ with

(i) $\mathbb{L}^{k}=\left(\mathrm{v}_{1}, \ldots, \mathrm{v}_{k}\right) \mathbb{Z}^{k}$,

(ii) ${ }^{T} \mathbb{L}^{n-k}=\left(\mathrm{v}_{k+1}, \ldots, \mathrm{v}_{n}\right) \mathbb{Z}^{n-k}$,

(iii) there is an $x \in \mathscr{F}^{0}(\check{C})$ with $\left\{\mathrm{v}_{1}, \ldots, \mathrm{v}_{k}\right\} \subset \mathscr{F}^{0}(C+$ $x)$ where $\breve{C}$ is the reflection of the unit cell of $\mathbb{L}^{n}$ at the origin.

Condition (iii) ensures that integration over 'local knowledge' on the image data is possible as needed later. The translative complement ${ }^{T} \mathbb{L}^{n-k}$ has properties similar to those of the orthogonal complement of a linear subspace. In particular, the union of shifts $\mathbb{L}^{k}+x$ of the section lattice over all $x$ from the corresponding translation lattice ${ }^{T} \mathbb{L}^{n-k}$ yields the cubic lattice $\mathbb{L}^{n}=$ $\bigcup_{x \in T} \mathbb{L}^{n-k} \mathbb{L}^{k}+x$. However, the translative complement is not necessarily uniquely determined. Nevertheless, choosing one of the translative complements arbitrarily turns out to cover all applications presented in the following.

\section{MEASUREMENT}

We denote by $\mathscr{L}^{k}$ the set of all $k$-dimensional linear subspaces of $\mathbb{R}^{n} ;{ }^{\perp} L$ is the orthogonal complement of $L \in \mathscr{L}^{k} ; V_{\perp_{L}}$ is the $n$-k-dimensional Lebesgue measure on ${ }^{\perp} L$, and $\mu$ denotes the rotation invariant measure on $\mathscr{L}^{k}$ with $\mu\left(\mathscr{L}^{k}\right)=1$. Now the Crofton formulae for the intrinsic volumes $V_{n-k}(X)$ of a compact and polyconvex set $X$ can be written in the form

$$
\begin{array}{r}
\alpha_{n 0 k} V_{n-k}(X) \\
=\int_{\mathscr{L}^{k}} \underbrace{\int_{\perp_{L}} \chi(X \cap(L+y)) V_{\perp_{L}}(d y)}_{p_{X}^{k}(L)} \mu(d L),
\end{array}
$$

$k=1, \ldots, n-1$, with the constants

$$
\alpha_{n 0 k}=\frac{\Gamma\left(\frac{k+1}{2}\right) \Gamma\left(\frac{n-k+1}{2}\right)}{\sqrt{\pi} \Gamma\left(\frac{n+1}{2}\right)} .
$$

This means that the intrinsic volumes can be expressed in terms of the Euler number measured on lowerdimensional subspaces.

The observation of $X$ on the lattice $\mathbb{L}^{n}$ implies that the integrand in the Crofton formulae (Eq. 9) is known for only a finite number of elements of $\mathscr{L}^{k}$ and the translation $L+y$ is possible for discrete values of $y$, only. That is, both integrals in Eq. 9 are approximated by sums. Furthermore, the intersection $X \cap(L+y)$ must be replaced by its discretisation $X \sqcap\left(\mathbb{F}^{k}+y\right)$ with respect to an adjacency system $\mathbb{F}^{k}$ in $\mathbb{L}^{k}$ where $L=\operatorname{span} \mathbb{L}^{k}$. Finally, the translations $y$ are from ${ }^{T} \mathbb{L}^{n-k}$ instead of ${ }^{\perp} L$, where ${ }^{T} \mathbb{L}^{n-k}$ is a translative complement according to Definition 4.

\section{DISCRETISATION OF THE TRANSLATIVE INTEGRAL}

For simplicity we restrict ourselves to the special case where $X$ is observed on a cubic primitive lattice. Nevertheless, this is not a substantial restriction and the following considerations can be extended to arbitrary homogeneous lattices, too.

Let $C^{k}$ and $\mathbb{F}^{k}$ be a lattice cell and an adjacency system of a section lattice $\mathbb{L}^{k}$ of $a \mathbb{Z}^{n}$, respectively. Denote by ${ }^{T} C^{n-k}$ a unit cell of the translation 
lattice ${ }^{T} \mathbb{L}^{n-k}$ of $\mathbb{L}^{k}$ (according Definition 4 ) and by proj ${ }^{T} C^{n-k}$ the orthogonal projection of ${ }^{T} C^{n-k}$ onto ${ }^{\perp} L={ }^{\perp}\left(\operatorname{span} \mathbb{L}^{k}\right)$. Denote its volume by $\operatorname{vp}_{n-k}:=$ vol proj ${ }^{T} C^{n-k}$ and note that the ratio $\mathrm{vp}_{n-k} / a^{n-k}$ is an unscaled quantity. We have

$$
\operatorname{vp}_{n-k}=\operatorname{vol} \operatorname{proj}^{T} C^{n-k}=\frac{\operatorname{vol} C}{\operatorname{vol} C^{k}}, \quad k=1, \ldots, n-1 .
$$

Now, in analogy to the rectangular quadrature rule, the translative (inner) integral in Eq. 9 can be approximated by

$$
\begin{aligned}
& p_{X}^{k}(L) \approx \operatorname{vp}_{n-k} \sum_{y \in T^{n-k}} \chi((X-y) \cap L) \\
& \approx \operatorname{vp}_{n-k} \sum_{y \in \mathbb{L}^{n-k}} \chi\left((X-y) \sqcap \mathbb{F}^{k}\right) \\
& \stackrel{(6)}{=} \operatorname{vp}_{n-k} \sum_{y \in \mathbb{L}^{n-k}} \sum_{z \in \mathbb{L}^{k}} \chi_{0}^{k}\left(C^{k} \cap\left(\left((X-y) \sqcap \mathbb{F}^{k}\right)-z\right)\right) \\
& =\operatorname{vp}_{n-k} \sum_{x \in \mathbb{L}^{n}} \chi_{0}^{k}\left(C^{k} \cap\left((X-x) \sqcap \mathbb{F}^{k}\right)\right),
\end{aligned}
$$

where the local Euler number $\chi_{0}^{k}$ is defined as in Eq. 5 but w. r. t. the adjacency system $\mathbb{F}^{k}$ on the section lattice $\mathbb{L}^{k}$.

The last expression can be considered as an estimator $\hat{p}_{X}^{k}(L)$ of the translative integral $p_{X}^{k}(L)$. For a fixed section lattice $\mathbb{L}^{k}$, the volume of $\operatorname{proj}^{T} C^{n-k}$ and thus $\hat{p}_{X}^{k}(L)$ do not depend on the particular choice of ${ }^{T} \mathbb{L}^{n-k}$. This follows directly from Eq. 10 and the definition of the $\mathbb{L}^{k}$, see Definition 4 .

It can be seen that the right-hand side of Eq. 11 is a scalar product,

$$
\begin{aligned}
\hat{p}_{X}^{k}(L) & =\sum_{\ell=0}^{v_{k}} \underbrace{\operatorname{vp}_{n-k} \chi_{0}^{k}\left(\zeta_{\ell} \sqcap \mathbb{F}^{k}\right)}_{a^{n-k} \tilde{w}_{\ell}^{(k)}} . \\
& \underbrace{\sum_{x \in \mathbb{L}^{n}} \mathbf{1}\left(\zeta_{\ell}+x \subseteq X\right) \mathbf{1}\left(\zeta_{v_{k}-\ell}+x \subseteq X^{c}\right)}_{h_{\ell}^{(k)}},
\end{aligned}
$$

with $v_{k}=2^{2^{k}}-1$ and the local pixel configurations $\zeta_{\ell} \subseteq \mathscr{F}^{0}\left(C^{k}\right)$ on $\mathbb{L}^{k}$, where the indexing of the $\zeta_{\ell}$ is chosen analogously to the one for the $\xi_{\ell}$. Notice that the vector $\tilde{w}^{(k)}=\left(\tilde{w}_{\ell}^{(k)}\right)$ depends on the adjacency system $\mathbb{F}^{k}$ and the vector $h^{(k)}=\left(h_{\ell}^{(k)}\right)$ depends on the sample $X \cap \mathbb{L}^{k}$ of $X$ on $L^{k}$.

The computation of the vectors $h^{(k)}$ involves access to all pixel values of the image. Therefore, this is the most time consuming part in the estimation of the translative integrals in Eq. 9. Thus, from the algorithmic point of view the following question arises: Is it possible to compute all translative integrals from the same $h$ determined just once for a given set $X$ and lattice $\mathbb{L}^{n}$ ? In order to use this one $h$ for all dimensions $k$ and all section lattices $\mathbb{L}^{k}$, the vectors $\tilde{w}^{(k)}$ of weights have to be adapted, such that the scalar product $\tilde{w}^{(k)} h^{(k)}$ has a representation as $w^{(k)} h$. In order to achieve this, we consider the following two cases:

(i) Due to Definition 4(iii), the section lattice $\mathbb{L}^{k}$ has the property that there is a translation $y \in \mathbb{L}^{n}$ such that $C^{k}+y \subset C$. Then it follows that $C^{k}=(C-y) \cap L$ and

$$
\begin{aligned}
\chi_{0}^{k}\left(C^{k} \cap\left((X-x) \sqcap \mathbb{F}^{k}\right)\right) & = \\
\chi_{0}^{k}((C-y) & \left.\cap\left((X-x) \sqcap \mathbb{F}^{k}\right)\right),
\end{aligned}
$$

for all $x \in \mathbb{L}^{n}$. This yields

$$
\hat{p}_{X}^{k}(L)=\operatorname{vp}_{n-k} \sum_{x \in \mathbb{L}^{n}} \chi_{0}^{k}\left((C-y) \cap\left((X-x) \sqcap \mathbb{F}^{k}\right)\right) .
$$

From Eq. 5 we obtain

$$
\chi_{0}^{k}\left(\zeta_{j} \sqcap \mathbb{F}^{k}\right)=\chi_{0}^{k}\left(\left(\xi_{\ell}-y\right) \sqcap \mathbb{F}^{k}\right)
$$

for all $j \in\left\{0, \ldots, v_{k}\right\}$ and all $\ell \in\{0, \ldots, v\}$ with $\zeta_{j} \subseteq \xi_{\ell}-y$ and $\zeta_{v_{k}-j} \subseteq \xi_{v-\ell}-y$. Furthermore, one gets

$$
h_{j}^{(k)}=\sum_{\ell=0}^{v} h_{\ell} \mathbf{1}\left(\zeta_{j} \subseteq \xi_{\ell}-y, \zeta_{v_{k}-j} \subseteq \xi_{v-\ell}-y\right),
$$

for $j=0, \ldots, v_{k}$. Using this, Eq. 12 can be rewritten as

$$
\begin{aligned}
\hat{p}_{X}^{k}(L)= & \sum_{\ell=0}^{v} \underbrace{\mathrm{vp}_{n-k} \chi_{0}^{k}\left(\left(\xi_{\ell}-y\right) \sqcap \mathbb{F}^{k}\right)}_{a^{n-k} w_{\ell}^{(k)}} \\
& \cdot \underbrace{\sum_{x \in \mathbb{L}^{n}} \mathbf{1}\left(\xi_{\ell}+x \subseteq X\right) \mathbf{1}\left(\xi_{v-\ell}+x \subseteq X^{c}\right)}_{h_{\ell}},
\end{aligned}
$$

defining the vector $w^{(k)}=\left(w_{\ell}^{(k)}\right)$.

(ii) Now we treat those section lattices having the property that there is no translation $y \in \mathbb{L}^{n}$ with $C^{k}+y \subset C$. Then Definition 4(iii) ensures that there are finitely many $x \in \mathbb{L}^{n}$ such that the intersections $C^{k} \cap(C-x)$ generate a face-to-face tessellation of $C^{k}$. This tessellation can be refined into a tessellation $\mathscr{G}_{0}=\left\{P_{1}, \ldots, P_{m}\right\}$ of $C^{k}$ into $k$ simplices $P_{i}$ such that for each $P_{i}$ there is a $y_{i} \in$ $\mathbb{L}^{n}$ with $P_{i}+y_{i} \in C$ and $\mathscr{F}^{0}\left(P_{i}+y_{i}\right) \subset \mathscr{F}^{0}(C)$. 
Then the adjacency system $\mathbb{F}^{k}$ is generated by the tessellation $\mathscr{G}_{0}$. Making use of Eq. 7, it can be shown that $\hat{p}_{X}^{k}(L)$ has the same form as above but with the weights

$$
\begin{aligned}
w_{\ell}^{(k)} & =\frac{\mathrm{vp}_{n-k}}{a^{n-k}} \sum_{i=1}^{m} \sum_{j=0}^{n}(-1)^{j} \\
& \cdot \sum_{F \in \mathscr{F} j\left(P_{i}\right)} \frac{1}{\tilde{\mu}_{F}} \mathbf{1}\left(\mathscr{F}^{0}(F) \subseteq\left(\xi_{\ell} \cap\left(P_{i}+y_{i}\right)\right)\right)
\end{aligned}
$$

for $\ell=0, \ldots, v$.

Summarising, we have shown that for all dimensions $k$ and for all section lattices $\mathbb{L}^{k}$, the translative integrals $p_{X}^{k}(L)$ in the Crofton formulae (Eq. 9) can be estimated via

$$
\hat{p}_{X}^{k}(L)=a^{n-k} \sum_{\ell=0}^{v} w_{\ell}^{(k)} h_{\ell}, \quad k=1, \ldots, n-1,
$$

where $L=\operatorname{span} \mathbb{L}^{k}$ is the corresponding subspace.

\section{DISCRETISATION OF THE INTEGRAL OVER ALL SUBSPACES}

From the $\mathbb{L}^{n}$-sampling of $X$ the estimator $\hat{p}_{X}^{k}\left(L_{i}\right)$ of the translative integral of Eq. 9 is known for finitely many subspaces $L_{i}=\operatorname{span} \mathbb{L}_{i}^{k}, i=1, \ldots, m_{k}$, only. Hence, one has to find an appropriate approximation of $\int_{\mathscr{L}^{k}} p_{X}^{k}(L) d \mu(L)$.

Applying a simple quadrature yields

$$
\begin{aligned}
\int_{\mathscr{L}^{k}} p_{X}^{k}(L) \mathrm{d} \mu(L) & \approx \int_{\mathscr{L}^{k}} \hat{p}_{X}^{k}(L) \mathrm{d} \mu(L) \\
& \approx \sum_{i=1}^{m_{k}} \gamma_{i}^{(k)} \hat{p}_{X}^{k}\left(L_{i}\right),
\end{aligned}
$$

where $\gamma_{i}^{(k)}$ are suitable weights. The choice of these weights is not trivial since the planes $L_{1}, \ldots, L_{m_{k}}$ are not uniformly scattered in $\mathscr{L}^{k}$. Moreover, the estimates $\hat{p}_{X}^{k}\left(L_{i}\right)$ of the $p_{X}^{k}\left(L_{i}\right)$ are not of the same accuracy for different subspaces. In $2 \mathrm{D}$, the $2 \pi$-periodic rectangle rule leads to weights which are proportional to the lengths of the Voronoï arcs corresponding to directions of the straight lines $L_{i}$. This geometric interpretation can be extended to higher dimensions which leads to a generalisation of the rectangular rule. For the special case of a cubic primitive lattice $\mathbb{L}^{3}=a \mathbb{Z}^{3}, a>0$, we have $\gamma_{i}^{(1)}=\gamma_{i}^{(2)}$ for all $i$, and the numerical values are $\gamma_{i}^{(k)}=0.091556$ for $i=1,2,3 ; \gamma_{i}^{(k)}=0.073961$ for $i=4, \ldots, 9 ; \gamma_{i}^{(k)}=0.070391$ for $i=10, \ldots, 13$, see Lang et al. (2001).
Summarising formulae Eqs. 9, 13 and 14, we obtain the approximation $\hat{V}_{n-k}(X)$ of $V_{n-k}(X)$ as

$$
\begin{aligned}
\hat{V}_{n-k}(X) & =\frac{1}{\alpha_{n 0 k}} \sum_{i=1}^{m_{k}} \gamma_{i}^{(k)} a^{n-k} \sum_{\ell=0}^{v} w_{\ell}^{(i, k)} h_{\ell} \\
& =\frac{a^{n-k}}{\alpha_{n 0 k}} \sum_{\ell=0}^{v} \underbrace{\sum_{i=1}^{m_{k}} \gamma_{i}^{(k)} w_{\ell}^{(i, k)}}_{v_{\ell}^{(k)}} h_{\ell},
\end{aligned}
$$

where the $w_{\ell}^{(i, k)}$ are the coefficients corresponding to the adjacency systems $\mathbb{F}_{i}^{k}$ on the subspaces $L_{i}$ according to Eq. 13.

Table 1. Surface area weights $4 v_{\ell}^{(1)}$, weights $2 \pi v_{\ell}^{(2)}$ for computing the integral of the mean curvature and the weights $v^{(3)}$ for the Euler number. The weights for the Euler number are given for the pairs $\left(\mathbb{F}_{6}, \mathbb{F}_{26}\right)$,

\begin{tabular}{|c|c|c|c|c|c|}
\hline$j$ & $\eta_{j}$ & & $4 v_{\ell}^{(1)}$ & $2 \pi v_{\ell}^{(2)}$ & $8 v_{\ell}^{(3)}$ \\
\hline 0 & $\xi_{0}$ & ?ी & 0 & 0 & $\begin{array}{llll}0 & 0 & 0 & 0\end{array}$ \\
\hline 1 & $\xi_{1}$ & ? & 0.376 & 0.590 & $\begin{array}{llll}1 & 1 & 1 & 1\end{array}$ \\
\hline 2 & $\xi_{3}$ & III & 0.659 & 0.728 & $\begin{array}{llll}0 & 0 & 0 & 0\end{array}$ \\
\hline 3 & $\xi_{0}$ & ! & 0.646 & 0.616 & $\begin{array}{llll}2 & 0 & 0 & -2\end{array}$ \\
\hline 4 & $\xi_{129}$ & 过 & 0.588 & 0.687 & $\begin{array}{llll}2 & 0 & 0 & -6\end{array}$ \\
\hline 5 & $\xi_{7}$ & [?] & 0.839 & 0.446 & $-1-1-1-1$ \\
\hline 6 & $\xi_{131}$ & ifit. & 0.768 & 0.426 & $1-1-1-3$ \\
\hline 7 & $\xi_{41}$ & ?III & 0.813 & 0.3 & $3-3-1-1$ \\
\hline 8 & $\xi_{15}$ & ?f? & 927 & 0 & $\begin{array}{llll}0 & 0 & 0 & 0\end{array}$ \\
\hline 9 & $\xi_{43}$ & 通 & 0.914 & 0 & $-2-2-2-2$ \\
\hline 10 & $\xi_{139}$ & 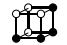 & 0.856 & 0 & $-2-2-2-2$ \\
\hline 11 & $\xi_{153}$ & \pm & 0.785 & 0 & $\begin{array}{llll}0 & 0 & -2 & 0\end{array}$ \\
\hline 12 & $\xi_{105}$ & tin & 0.874 & 0 & $\begin{array}{llll}4-8 & 0 & 4\end{array}$ \\
\hline 13 & $\xi_{99}$ & II & 0.843 & 0 & $0-2-2 \quad 0$ \\
\hline 14 & $\xi_{214}$ & tid & 0.813 & -0.3 & $\begin{array}{llll}-1 & -3 & -1 & 3\end{array}$ \\
\hline 1. & $\xi_{124}$ & II & 0.768 & -0.426 & $\begin{array}{llll}-3 & -1 & -1 & 1\end{array}$ \\
\hline 16 & $\xi_{248}$ & ti़ी & 0.839 & -0.446 & $-1-1-1-1$ \\
\hline 17 & $\xi_{126}$ & 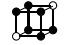 & 0.588 & -0.687 & $\begin{array}{llll}-6 & 0 & 0 & 2\end{array}$ \\
\hline 18 & $\xi_{246}$ & II & 0.646 & -0.616 & $\begin{array}{llll}-2 & 0 & 0 & 2\end{array}$ \\
\hline 19 & $\xi_{252}$ & tتी & 0.659 & -0.728 & $\begin{array}{llll}0 & 0 & 0 & 0\end{array}$ \\
\hline 20 & $\xi_{254}$ & 10. & 0.376 & -0.590 & $\begin{array}{llll}1 & 1 & 1 & 1\end{array}$ \\
\hline 21 & $\xi_{255}$ & & 0 & 0 & $\begin{array}{llll}0 & 0 & 0 & 0\end{array}$ \\
\hline
\end{tabular}
$\left(\mathbb{F}_{14.1}, \mathbb{F}_{14.1}\right),\left(\mathbb{F}_{14.2}, \mathbb{F}_{14.2}\right)$ and $\left(\mathbb{F}_{26}, \mathbb{F}_{6}\right)$ (from left to right). The table shows the values for $8 v^{(3)}$ for ease of reading. The weights for $\left(\mathbb{F}_{14.1}, \mathbb{F}_{14.1}\right)$ and $\left(\mathbb{F}_{14.2}, \mathbb{F}_{14.2}\right)$ are averages over the transforms in $\mathscr{M}$ as the adjacency systems $\mathbb{F}_{14.1}$ and $\mathbb{F}_{14.1}$ are not isotropic (i.e., invariant w. r. t. the linear transforms in $\mathscr{M}$ ). 
The last equation shows that estimates of the intrinsic volumes $V_{n-k}(X)$ can also be written as

$$
\hat{V}_{n-k}(X)=\frac{a^{n-k}}{\alpha_{n 0 k}} v^{(k)} h, \quad k=1, \ldots, n-1,
$$

with the scalar products $v^{(k)} h$ of the vectors $v^{(k)}=$ $\left(v_{\ell}^{(k)}\right)$ and $h$. We complete the above set of estimators by those of the volume $V_{n}$ and the Euler number $V_{0}$ of $X$ :

$$
\hat{V}_{n}(X)=a^{n} v^{(0)} h,
$$

with the coefficients $v_{\ell}^{(0)}=\operatorname{vol} C / a^{n}$ for odd $\ell$, and $v_{\ell}^{(0)}=0$ otherwise;

$$
\hat{V}_{0}(X)=v^{(n)} h,
$$

with the coefficients $v_{\ell}^{(n)}:=w_{\ell}=\chi_{0}^{n}\left(\xi_{\ell} \sqcap \mathbb{F}\right)$ where $\mathbb{F}$ is an adjacency system on $\mathbb{L}^{n}$.

For $n=3$ we get $\alpha_{301}=\alpha_{302}=\frac{1}{2}$. The intrinsic volumes $V_{2}$ and $V_{1}$, and thus the surface area $S$ and the integral of the mean curvature $M$, can be estimated as

$$
\begin{aligned}
\hat{S}(X) & =2 \hat{V}_{2}(X)=4 a^{2} v^{(1)} h, \\
\hat{M}(X) & =\pi \hat{V}_{1}(X)=2 \pi a v^{(2)} h .
\end{aligned}
$$

The surface area weights $4 v^{(1)}$, also published in Schladitz et al. (2006), the weights $2 \pi v^{(2)}$ for the integral of mean curvature and the weights $v^{(3)}$ for the Euler number are presented in Table 1. The weights for the Euler number were first published in Ohser et al. (2002).

The surface area and the integral of mean curvature are rotation invariant functionals. Thus the weights can be presented for the representatives $\eta_{j}$ of the congruence classes $D_{j}$ of the pixel configurations $\xi_{0}, \ldots, \xi_{255}$. Notice that for the surface area the weights for complementary representatives $\eta_{j}^{c}$ are the same as for $\eta_{j}$ while in the case of the integral of mean curvature the sign switches.

\section{INTRINSIC VOLUME DENSITIES}

Instead of deterministic sets we consider now random sets widely used as geometric models for constituents of microstructures. The characterisation of a constituent simplifies considerably in case of macroscopic homogeneity and even further if the microstructure is isotropic, too. In these cases, the intrinsic volume densities are important geometric characteristics of the constituents.
In principle, the intrinsic volume densities of a constituent can be estimated by the intrinsic volumes related to the volume of the observation window, see Chapter 4 in Ohser and Mücklich (2000) and Lang et al. (2001). Alternative approaches Klenk et al. (2006), Mrkvička and Rataj (2008), Schmidt and Spodarev (2005) are based on solutions of systems of linear equations deduced from the local Steiner formula, see Section 4.4 in Schneider (1993), or the principal kinematic formula. These methods are studied in detail for the 2-dimensional case but work in principle in arbitrary dimensions. Comparisons for Boolean models in 2D in Guderlei et al. (2007) and Mrkvička and Rataj (2008) show, that the accuracy of the resulting estimators is sometimes higher than of those given in Chapter 4 of Ohser and Mücklich (2000) using the present approach. However, so far none of these algorithms has been proved to work in practice in dimensions $n \geq 3$.

\section{MACROSCOPIC HOMOGENEITY}

Let $\Xi$ be a macroscopically homogeneous random set on $\mathbb{R}^{n}$ observed in a compact and convex window $W$ with nonempty interior. Assume now that the realisations of $\Xi$ belong to the extended convex ring almost surely and thus their intersections with $W$ are polyconvex sets. Furthermore, assume that $\Xi$ fulfils the integrability condition $\mathbb{E} 2^{\#(\Xi \cap K)}<\infty$ for any compact and convex set $K$, where $\# X$ denotes the minimal number $m$ such that the set $X$ has a representation $X=$ $K_{1} \cup \ldots \cup K_{m}$ with compact and convex sets $K_{1}, \ldots, K_{m}$. Then the intrinsic volume densities $V_{V, k}$ of $\Xi$ exist and can be defined by the limits

$$
V_{V, k}(\Xi)=\lim _{r \rightarrow \infty} \frac{\mathbb{E} V_{k}(\Xi \cap r W)}{\operatorname{vol}(r W)}, \quad k=0, \ldots, n,
$$

see, e.g., Schneider and Weil (2008), p. 398.

The random set $\Xi$ may be observed on the homogeneous lattice $\mathbb{L}^{n}$ with the unit cell $C$. By $\Xi \cap \mathbb{L}^{n}$ we denote the $\mathbb{L}^{n}$-sampling of $\Xi$ where $\mathbb{L}^{n}$ is a equipped with a pair $\left(\mathbb{F}, \mathbb{F}_{c}\right)$ of complementary adjacency systems. Furthermore, as in Section 4.4 the section lattices $\mathbb{L}_{i}^{k}$ of $\mathbb{L}^{n}$ are equipped with pairs $\left(\mathbb{F}_{i}^{k}, \mathbb{F}_{c, i}^{k}\right)$ of complementary adjacency systems, $i=$ $1, \ldots, m_{k}, k=1, \ldots, n-1$. Again, for the sake of simplicity we concentrate on samplings on cubic primitive lattices.

Now the method described in Section 4.2 is adapted to the measurement of the volume density. We choose a window $W$ such that $\mathbb{L}^{n} \cap(W \ominus \check{C}) \neq \emptyset$, where $W \ominus \breve{C}$ is the window $W$ reduced by the reflection of the unit cell $C$ of $\mathbb{L}^{n}$. Furthermore, let $\tilde{h}=\left(\tilde{h}_{\ell}\right)$ 
be the vector of numbers of local configurations in $\Xi \cap W \cap \mathbb{L}^{n}$,

$$
\tilde{h}_{\ell}=\sum_{x \in \mathbb{L}^{n} \cap(W \ominus \check{C})} \mathbf{1}\left(\xi_{\ell}+x \subset \Xi\right) \mathbf{1}\left(\xi_{v-\ell}+x \subset \Xi^{c}\right),
$$

for $\ell=0, \ldots, v$. Then estimators of the intrinsic volumes $V_{V, n-k}$ can be given by

$$
\tilde{V}_{V, n-k}(\Xi)=\frac{a^{n-k} v^{(k)} \tilde{h}}{\alpha_{n 0 k} \#\left(\mathbb{L}^{n} \cap(W \ominus \check{C})\right) \operatorname{vol} C},
$$

for $k=0, \ldots, n$. These estimators are usually biased for $k>0$. Because of the macroscopic homogeneity of $\Xi$, the expectations of the $\tilde{V}_{V, n-k}$ depend on the probabilities that the local configurations $\xi_{\ell}$ belong to the foreground $\Xi$ and the complementary configurations $\xi_{v-\ell}$ are in the background $\Xi^{c}$. From $\operatorname{vol} C=a^{n}$ and $\mathbb{E} \tilde{h}_{\ell} / \#\left(\mathbb{L}^{n} \cap(W \ominus \check{C})\right)=\mathbb{P}\left(\xi_{\ell} \subseteq\right.$ $\left.\Xi, \xi_{v-\ell} \subseteq \Xi^{c}\right)$ it follows that

$$
\mathbb{E} \tilde{V}_{V, n-k}(\Xi)=\frac{1}{\alpha_{n 0 k} a^{k}} \sum_{\ell=0}^{v} v_{\ell}^{(k)} \mathbb{P}\left(\xi_{\ell} \subseteq \Xi, \xi_{v-\ell} \subseteq \Xi^{c}\right),
$$

for $k=0, \ldots, n$.

In order to assess estimation errors in the theoretical considerations below, the above probabilities are expressed in terms of $\mathbb{P}\left(\xi_{\ell} \subseteq \Xi^{c}\right)$. For each local configuration $\xi_{\ell}$ and a point $x \in \xi_{\ell}$ one gets

$$
\begin{aligned}
\mathbb{P}\left(\xi_{\ell} \subseteq \Xi, \xi_{v-\ell} \subseteq \Xi^{c}\right) \\
=\quad \mathbb{P}\left(\xi_{\ell} \backslash\{x\} \subseteq \Xi, \xi_{v-\ell} \subseteq \Xi^{c}\right) \\
\quad-\mathbb{P}\left(\xi_{\ell} \backslash\{x\} \subseteq \Xi, \xi_{v-\ell} \cup\{x\} \subseteq \Xi^{c}\right) .
\end{aligned}
$$

Recursion yields the linear equation system

$$
\mathbb{P}\left(\xi_{\ell} \subseteq \Xi, \xi_{v-\ell} \subseteq \Xi^{c}\right)=\sum_{j=0}^{v} b_{j \ell} \mathbb{P}\left(\xi_{j} \subseteq \Xi^{c}\right),
$$

for $\ell=0, \ldots, v$, where the $b_{j \ell}$ are integers. (We remark that Eq. 18 as well as the explicit values of the $b_{j \ell}$ can be derived from the inclusion-exclusion formula, too.) Plugging (18) into (17) we obtain

$$
\begin{gathered}
\mathbb{E} \tilde{V}_{V, n-k}(\Xi)=\frac{1}{\alpha_{n 0 k} a^{k}} \sum_{\ell=0}^{v} v_{\ell}^{(k)} \sum_{j=0}^{v} b_{j \ell} \mathbb{P}\left(\xi_{j} \subseteq \Xi^{c}\right) \\
=\frac{1}{\alpha_{n 0 k} a^{k}} \sum_{j=0}^{\sum_{\ell=0}^{\sum_{\ell} v_{\ell}^{(k)} b_{j \ell}}} \mathbb{P}\left(\xi_{j} \subseteq \Xi^{c}\right), \quad \text { (19) } \\
g_{j}^{(k)}
\end{gathered}
$$

\begin{tabular}{|c|c|c|c|c|c|c|c|}
\hline$j$ & $\eta_{j}$ & $\bar{g}_{j}^{(1)}$ & $\bar{g}_{j}^{(2)}$ & \multicolumn{4}{|c|}{$8 \bar{g}_{j}^{(3)}$} \\
\hline 0 & $\xi_{0}$ 面 & 0 & 0 & 0 & 0 & 0 & 0 \\
\hline 1 & $\xi_{1}$ 而 & 0.751 & 0.751 & 1 & 1 & 1 & 1 \\
\hline 2 & $\xi_{3} \rightleftarrows$ & -0.275 & -0.861 & -3 & -3 & -3 & -3 \\
\hline 3 & $\xi_{9}$ 且 & -0.314 & -1.076 & & -3 & -3 & -6 \\
\hline 4 & $\xi_{129}$ & -0.163 & -0.314 & & -1 & -1 & -4 \\
\hline 5 & $\xi_{11}$ & 0 & 0.549 & 0 & 6 & 6 & 12 \\
\hline 6 & $\xi_{131}$ 跑 & 0 & 0.628 & 0 & 6 & 4 & 24 \\
\hline 7 & $\xi_{41}$ & 0 & 0.325 & 0 & 0 & 2 & 8 \\
\hline 8 & $\xi_{15}$ & 0 & 0 & 3 & 0 & 0 & -3 \\
\hline 9 & $\xi_{43}$ & 0 & 0 & 0 & & -2 & -8 \\
\hline 10 & $\xi_{139}$ & 0 & 0 & 0 & & & -24 \\
\hline 11 & $\xi_{159}$ & 0 & 0 & 0 & 0 & 0 & \\
\hline 12 & $\xi_{105}$ & 0 & 0 & 0 & 0 & 0 & -2 \\
\hline 13 & $\xi_{99}$ & 0 & 0 & 0 & 0 & & -24 \\
\hline 14 & $\xi_{214}$ & 0 & 0 & 0 & 0 & 0 & 8 \\
\hline 15 & $\xi_{124}$ & 0 & 0 & 0 & 0 & 0 & 24 \\
\hline 16 & $\xi_{248}$ & 0 & 0 & 0 & 0 & 0 & \\
\hline 17 & $\xi_{126}$ & 0 & 0 & 0 & 0 & & -4 \\
\hline 18 & $\xi_{246}$ & 0 & 0 & 0 & 0 & & -12 \\
\hline 19 & $\xi_{252}$ & 0 & 0 & 0 & 0 & & -12 \\
\hline 20 & $\xi_{254}$ & 0 & 0 & 0 & 0 & 0 & 8 \\
\hline 21 & $\xi_{255}$ & 0 & 0 & -1 & 0 & & -1 \\
\hline
\end{tabular}

for $k=0, \ldots, n$, where the weights $g_{j}^{(k)}$ are independent of $\Xi$.
Table 2. The weights $\bar{g}_{j}^{(k)}$ for the 22 congruence classes of the local pixel configurations in 3D-images. The first, second, third, fourth column of $\bar{g}_{i}^{(0)}$ correspond to the pairs $\left(\mathbb{F}_{26}, \mathbb{F}_{6}\right),\left(\mathbb{F}_{14.1}, \mathbb{F}_{14.1}\right),\left(\mathbb{F}_{14.2}, \mathbb{F}_{14.2}\right)$, and $\left(\mathbb{F}_{6}, \mathbb{F}_{26}\right)$, respectively.

\section{MACROSCOPIC HOMOGENEITY AND ISOTROPY}

Let now $\Xi$ be macroscopically homogeneous, isotropic and the distribution of $\Xi$ is invariant under reflection at the origin. For cubic primitive lattices it is sufficient to restrict the following considerations to the congruence classes $D_{j}$ of the local pixel configurations. Then the probabilities in Eq. 17 can be rewritten as

$$
\mathbb{P}\left(\xi_{\ell} \subseteq \Xi, \xi_{v-\ell} \subseteq \Xi^{c}\right)=\mathbb{P}\left(\eta_{j} \subseteq \Xi, \eta_{j}^{c} \subseteq \Xi^{c}\right),
$$

for all $\xi_{\ell} \in D_{j}$ and the linear equation system (18) takes the form

$$
\mathbb{P}\left(\eta_{\ell} \subseteq \Xi, \eta_{\ell}^{c} \subseteq \Xi^{c}\right)=\sum_{j=0}^{v_{0}} \bar{b}_{j \ell} \mathbb{P}\left(\eta_{j} \subseteq \Xi^{c}\right),
$$


for $\ell=0, \ldots, v_{0}$ and with integer coefficients $\bar{b}_{j \ell}$. Obviously, $\sum_{j=0}^{v_{0}} \mathbf{1}\left(\xi_{\ell} \in D_{j}\right)=1$ for all $\ell$ and, thus, the expectations of the intrinsic volumes can be expressed by

$$
\mathbb{E} \tilde{V}_{V, n-k}(\Xi)=\frac{1}{\alpha_{n 0 k} a^{k}} \sum_{j=0}^{v_{0}} \bar{g}_{j}^{(k)} \mathbb{P}\left(\eta_{j} \subseteq \Xi^{c}\right),
$$

with the coefficients

$$
\bar{g}_{i}^{(k)}=\sum_{j=0}^{v_{0}} \bar{b}_{i j} \sum_{\ell=0}^{v} v_{\ell}^{(k)} \mathbf{1}\left(\xi_{\ell} \in D_{j}\right)
$$

for $i=0, \ldots, v_{0}$ and $k=0, \ldots, n$.

Now we restrict the considerations to $\mathbb{R}^{3}$. Using the weights in the Table 1, appropriate estimators of the densities $S_{V}, M_{V}$ and $\chi_{V}$ of the surface area, the integral of the mean curvature and the Euler number are

$$
\tilde{S}_{V}=\frac{4 v^{(1)} \tilde{h}}{a \sum \tilde{h}_{\ell}}, \quad \tilde{M}_{V}=\frac{2 \pi v^{(2)} \tilde{h}}{a^{2} \sum \tilde{h}_{\ell}}, \quad \tilde{\chi}_{V}=\frac{v^{(3)} \tilde{h}}{a^{3} \sum \tilde{h}_{\ell}}
$$

respectively. Their expectations are

$$
\begin{aligned}
& \mathbb{E} \tilde{S}_{V}=\frac{4}{a} \sum_{j=0}^{21} \bar{g}_{j}^{(1)} \mathbb{P}\left(\eta_{j} \subseteq \Xi^{c}\right), \\
& \mathbb{E} \tilde{M}_{V}=\frac{2 \pi}{a^{2}} \sum_{j=0}^{21} \bar{g}_{j}^{(2)} \mathbb{P}\left(\eta_{j} \subseteq \Xi^{c}\right), \\
& \mathbb{E} \tilde{\chi}_{V}=\frac{1}{a^{3}} \sum_{j=0}^{21} \bar{g}_{j}^{(3)} \mathbb{P}\left(\eta_{j} \subseteq \Xi^{c}\right),
\end{aligned}
$$

respectively. The values of the $\bar{g}_{j}^{(k)}$ are listed in Table 2.

\section{INTRINSIC VOLUME DENSITIES OF BOOLEAN MODELS}

Now we consider Boolean models sampled on a cubic lattice $\mathbb{L}^{n}=a \mathbb{Z}^{n}, a>0$. In this section we present relationships between the expectations of the intrinsic volume densities of sampled Boolean models, the density of the germs and the corresponding expectations of the intrinsic volumes of the grains. Following Serra (1982) p. 492ff and p. 557, we derive formulae for $\mathbb{L}^{n}$-samplings of Boolean models connecting the parameters of the Boolean models with the expectations of the estimators $\tilde{V}_{V, k}$ of the densities of the intrinsic volumes. Comparisons with Miles' formulae yields the asymptotic bias of the estimators.

\section{BOOLEAN MODELS IN $\mathbb{R}^{n}$}

Let $\Phi=\left\{x_{1}, x_{2}, \ldots\right\}$ denote a macroscopically homogeneous Poisson point field in $\mathbb{R}^{n}$ (the point field of germs) with point density $\lambda>0$ and $\Xi_{1}, \Xi_{2}, \ldots$ a sequence of independent and identically distributed (i. i. d.) random, compact and convex sets (the grains) with nonempty interior and independent of $\Phi$. The corresponding Boolean model is defined as the random closed set

$$
\Xi=\bigcup_{j=1}^{\infty}\left(\Xi_{j}+x_{j}\right) .
$$

For more detailed definitions and explanations see Matheron (1975); Schneider and Weil (2008); Molchanov (1997). In the following we assume that the random grains $\Xi_{i}$ are isotropic and invariant w.r.t. reflection at the origin. Then $\Xi$ is isotropic, too, and invariant w.r.t. reflection at the origin. Moreover, assume $\mathbb{E} V_{k}\left(\Xi_{1}\right)<\infty$ for $k=1, \ldots, n$.

With probability one the intersection of two grains is either empty or has nonempty interior. Then from the consistency relation for the Euler number and Crofton's intersection formulae (9) it follows that $V_{V, k}(\Xi)=(-1)^{n-k+1} V_{V, k}\left(\overline{\Xi^{c}}\right)$ for $k=0, \ldots, n-1$.

For Boolean models, the probabilities occurring on the right hand side of Eq. 20 can be written as

$$
\mathbb{P}\left(\eta_{\ell} \subset \Xi^{c}\right)=\mathrm{e}^{-\lambda \mathbb{E} V_{n}\left(\Xi_{1} \oplus \eta_{\ell}\right)}, \quad \ell=0, \ldots, v_{0} .
$$

The expectations of volumes occurring on the right hand side of this equation can be calculated approximately by the Steiner formula when substituting $\eta_{\ell}$ with its convex hull $F_{\ell}=$ conv $\eta_{\ell}$. We can assume $V_{n}\left(\Xi_{1} \oplus \eta_{\ell}\right) \approx V_{n}\left(\Xi_{1} \oplus F_{\ell}\right)$ which follows for particular cases from the following lemmas.

Lemma 1 Let $K$ be a compact and convex set with nonempty interior, then

$$
V_{n}(K \oplus[0, a u])-V_{n}(K \oplus\{0, a u\})=o(a), u \in S^{n-1}
$$

as $a \downarrow 0$.

Proof For the Minkowski addition of $K$ with the segment $[0, a u], a>0$, we have

$$
V_{n}(K \oplus[0, a u])=V_{n}(K)+a V_{n-1}\left(\Pi\left(K,{ }^{\perp} \operatorname{span} u\right)\right),
$$

where $\Pi\left(K,{ }^{\perp} \operatorname{span} u\right)$ is the orthogonal projection of $K$ onto the $n$-1-dimensional subspace ${ }^{{ }^{\perp}} \operatorname{span} u$ orthogonal to the straight line spanned by $u$. On the other hand

$$
\begin{aligned}
V_{n}(K \oplus\{0, a u\}) & =V_{n}(K \cup(K+a u)) \\
& =2 V_{n}(K)-\operatorname{cov}(K, a u), \quad a \geq 0,
\end{aligned}
$$


where $\operatorname{cov}(K, a u)=V_{n}(K \cap(K+a u))$ denotes the covariogram function of the set $K$ w. r. t. $u$. Now, since $V(K)=\operatorname{cov}(K, 0)$ and

$$
V_{n-1}\left(\Pi\left(K,{ }^{\perp} \operatorname{span} u\right)\right)=-\left[\frac{d}{d t} \operatorname{cov}(K, t u)\right]_{t=0+},
$$

see Section 4.3 in Matheron (1975), it follows that

$$
\begin{aligned}
& \frac{V_{n}(K \oplus[0, a u])-V_{n}(K \oplus\{0, a u\})}{a} \\
& =\frac{\operatorname{cov}(K, a u)-\operatorname{cov}(K, 0)}{a}-\left[\frac{d}{d t} \operatorname{cov}(K, t u)\right]_{t=0+}
\end{aligned}
$$

and thus

$$
\lim _{a \downarrow 0} \frac{V_{n}(K \oplus[0, a u])-V_{n}(K \oplus\{0, a u\})}{a}=0 .
$$

We remark that Lemma 1 holds more generally for configurations $\xi \subseteq \mathscr{F}^{0}(C)$ and $F=\operatorname{conv} \xi$. This is a consequence of Corollary 2.(2) of Kiderlen and Rataj (2006) as the support function of a general nonempty compact set in $\mathbb{R}^{n}$ is defined as the support function of its convex hull.

Lemma 2 For $n \geq 3$, balls $B_{r}$ with fixed radius $r$, configurations $\xi \subseteq \mathscr{F}^{0}(C)$ and $F=$ conv $\xi$ with $\operatorname{dim} F \leq 2$ one gets

$$
V_{n}\left(B_{r} \oplus F\right)-V_{n}\left(B_{r} \oplus \xi\right)=o\left(a^{2}\right) \quad \text { as } \quad a \downarrow 0 .
$$

Proof First we observe that the distance between $\xi$ and $F$ is smaller than the space diagonal of the unit cell, $\max \{\|x-y\|: x \in F, y \in \xi\}<a \sqrt{n}$. For all $a<r / \sqrt{n}$ we can choose $t=\sqrt{r^{2}-n a^{2}}$. Then it follows that $F \oplus B_{t} \subset \xi \oplus B_{r} \subset F \oplus B_{r}$.

Now we replace $F \oplus B_{t}$ with a non-convex set having enlarged spherical sectors at the vertices of $F$. Let $H(F, u)$ be a supporting hyperplane of $F$ with normal direction $u$ and let $N(F, x)=\left\{u \in \mathbb{R}^{n} \backslash\{0\}: x \in\right.$ $H(F, u)\} \cup\{0\}$ denote the normal cone of $F$ at $x \in F$, see Schneider (1993), p. 70. Then $N(F, x) \cap B_{r}$ is the normal cone restricted to the ball $B_{r}$. The spherical sectors of $F$ at the vertices $x$ are given by $F_{r}(x)=$ $\left\{u+x: u \in N(F, x) \cap B_{r}\right\}$. Using this one obtains

$$
\left(F \oplus B_{t}\right) \cup \bigcup_{x \in \xi} F_{r}(x) \subset \xi \oplus B_{r} \subset F \oplus B_{r} .
$$

Since $\bigcup_{x \in \xi}\left(F_{r}(x)-x\right)=B_{r}$ and

$$
\left(\operatorname{int} F_{r}(x)-x\right) \cap\left(\operatorname{int} F_{r}(y)-y\right)=\emptyset,
$$

for all $x, y \in \xi$ with $x \neq y$, the Steiner formula implies that

$V_{n}\left(\left(F \oplus B_{t}\right) \cup \bigcup_{x \in \xi} F_{r}(x)\right)=\kappa_{n} r^{n}+\sum_{k=1}^{\operatorname{dim} F} \kappa_{n-k} t^{n-k} V_{k}(F)$.

Notice that since the intrinsic volumes $V_{j}$ are $j$ homogeneous, $V_{j}\left(\frac{F}{a}\right)=\frac{1}{a^{j}} V_{j}(F)$ for $a>0$, where the $V_{j}\left(\frac{F}{a}\right)$ are independent of the lattice spacing $a$. Now from Eq. 23, the $k$-homogeneity of $V_{k}$ and with the above choice for $t$ we get the estimation

$$
\begin{aligned}
0 & \leq V_{n}\left(B_{r} \oplus F\right)-V_{n}\left(B_{r} \oplus \xi\right) \\
& \leq V_{n}\left(B_{r} \oplus F\right)-V_{n}\left(\left(F \oplus B_{t}\right) \cup \bigcup_{x \in \xi} F_{r}(x)\right) \\
& \leq \sum_{k=1}^{\operatorname{dim} F} \kappa_{n-k}\left(r^{n-k}-t^{n-k}\right) V_{k}(F) \\
& =\sum_{k=1}^{\operatorname{dim} F} \kappa_{n-k} r^{n-k}\left(1-\left(1-\frac{n a^{2}}{r^{2}}\right)^{\frac{n-k}{2}}\right) a^{k} V_{k}\left(\frac{F}{a}\right) .
\end{aligned}
$$

Finally, the assertion of the above lemma follows from

$$
\lim _{a \downarrow 0} \frac{1-\left(1-\frac{n a^{2}}{r^{2}}\right)^{\frac{n-k}{2}}}{a^{2-k}}=0,
$$

as $n>2$, and $k>0$.

In order to estimate the error introduced by replacing $\eta_{\ell}$ with its convex hull $F_{\ell}=\operatorname{conv} \eta_{\ell}$ we have to derive lemmas similar to 1 and 2 but for the respective expectations.

Lemma 3 Let $X$ be a random compact and convex set whose distribution is isotropic and invariant w. r. t. reflection at the origin and whose interior is a. s. nonempty. Assume that $\mathbb{E} V_{n}(X)<\infty$ and that there is an $\varepsilon>0$ such that a. s. $B_{\varepsilon} \subseteq X$, then

$$
\mathbb{E}\left(V_{n}(X \oplus[0, a u])-V_{n}(X \oplus\{0, a u\})\right)=o(a),
$$

as $a \downarrow 0$ for all $u \in S^{n-1}$.

Proof Eqs. 21 and 22 imply

$$
\begin{aligned}
& V_{n}\left(X \oplus[0, a u]-V_{n}(X \oplus\{0, a u\})\right. \\
& \quad \leq a V_{n-1}\left(\Pi\left(X,{ }^{\perp} \operatorname{span} u\right)\right) \text { a. s. for all } u \in S^{n-1} .
\end{aligned}
$$

The assumptions $\mathbb{E} V_{n}(X)<\infty$ and $B_{\varepsilon} \subseteq X$ a. s. yield

$$
\mathbb{E} V_{n-1}(X)<n \frac{V_{n-1}\left(B_{\varepsilon}\right)}{V_{n}\left(B_{\varepsilon}\right)} \mathbb{E} V_{n}(X)<\infty,
$$


see Schneider (1993, p. 327), originally due to Wills (1970). Together with the convexity of $X$ this in turn ensures that $\mathbb{E} V_{n-1}\left(\Pi\left(X,{ }^{\perp} \operatorname{span} u\right)\right)<\infty$ for all $u \in S^{n-1}$ giving an integrable upper bound for the left hand side. This finally allows to interchange limit and expectation and the assertion follows from Lemma 1.

Lemma 4 For $n \geq 3$, let $B_{r} \subseteq \mathbb{R}^{n}$ be a ball of random diameter with $r \geq \varepsilon>0$ a. s. and $\mathbb{E} r^{n}<\infty$. Then for all configurations $\xi \subseteq \mathscr{F}^{0}(C)$ and $F=\operatorname{conv} \xi$ with $\operatorname{dim} F \leq 2$ one gets

$$
\mathbb{E}\left(V_{n}\left(B_{r} \oplus F\right)-V_{n}\left(B_{r} \oplus \xi\right)\right)=o\left(a^{2}\right) .
$$

Proof Eq. (24) and the assumption $\mathbb{E} r^{n}<\infty$ yield

$$
\begin{aligned}
& \mathbb{E}\left(V_{n}\left(B_{r} \oplus F\right)-V_{n}\left(B_{r} \oplus \xi\right)\right) \\
\leq & \sum_{k=1}^{\operatorname{dim} F} \kappa_{n-k} \mathbb{E} r^{n-k}\left(1-\left(1-\frac{n a^{2}}{\varepsilon^{2}}\right)^{\frac{n-k}{2}}\right) a^{k} V_{k}\left(\frac{F}{a}\right),
\end{aligned}
$$

and thus $\mathbb{E}\left(V_{n}\left(B_{r} \oplus F\right)-V_{n}\left(B_{r} \oplus \xi\right)\right)=o\left(a^{2}\right)$ using the same argument as in Lemma 2.

We remark that for every function $f: \mathbb{R} \mapsto \mathbb{R}$ with $f(a)=o\left(a^{m}\right)$ as $a \downarrow 0, m>0$, it follows that $1-\mathrm{e}^{f(a)}=$ $o\left(a^{m}\right)$.

As $F_{\ell}$ is convex, an application of the principal kinematic formula to macroscopic homogeneous Boolean models gives

$$
\begin{aligned}
\ln \mathbb{P}\left(F_{\ell} \subset \Xi^{c}\right) & =-\lambda \mathbb{E} V_{n}\left(\Xi_{1} \oplus F_{\ell}\right) \\
& =-\lambda \sum_{j=0}^{\operatorname{dim} F_{\ell}} \alpha_{n 0 j} V_{j}\left(F_{\ell}\right) \mathbb{E} V_{n-j}\left(\Xi_{1}\right),
\end{aligned}
$$

see Schneider and Weil (2008), p. 380. From the $j$ homogeneity of the intrinsic volumes $V_{j}$ it follows that the probability $\mathbb{P}\left(F_{\ell} \subset \Xi^{c}\right)$ can be considered as a function $f_{\ell}$ of the lattice distance $a$,

$$
f_{\ell}(a)=\exp \left\{-\lambda \sum_{j=0}^{\operatorname{dim} F_{\ell}} \alpha_{n 0 j} a^{j} V_{j}\left(\frac{F_{\ell}}{a}\right) \mathbb{E} V_{n-j}\left(\Xi_{1}\right)\right\},
$$

where the $V_{j}\left(\frac{F_{\ell}}{a}\right)$ are independent of $a$. Note that $f_{\ell}(a)=f_{\ell}(0)$ for $F_{\ell}$ with $\operatorname{dim} F_{\ell}=0$. For $F_{\ell}$ with $\operatorname{dim} F_{\ell}>0$ we now use a Taylor expansion of $f_{\ell}$,

$$
f_{\ell}(a)=\sum_{i=0}^{\operatorname{dim} F_{\ell}} \frac{f_{\ell}^{(i)}(0)}{i !} a^{i}+o\left(a^{\operatorname{dim} F_{\ell}}\right),
$$

where $f_{\ell}^{(i)}$ is the $i$ th derivative of $f_{\ell}$.

From the above approach and Eq. 20, the expectations of the estimators $\tilde{V}_{V, n-k}(\Xi)$ can be expressed in terms of the derivatives of the functions $f_{\ell}$. Using Lemma 3 and Lemma 4, respectively, we can formulate the following theorems.

Theorem 1 Let $\Xi$ be a homogeneous and isotropic Boolean model with random, compact, convex grains whose distribution is invariant w. r. t. reflection at the origin. If there is an $\varepsilon>0$ such that a. s. $B_{\varepsilon} \subseteq \Xi_{1}$ and $\mathbb{E} V_{n}\left(\Xi_{1}\right)<\infty$, then

$$
\mathbb{E} \tilde{V}_{V, n-1}(\Xi)=\frac{1}{\alpha_{n 01}} \sum_{\ell=0}^{v_{0}} \bar{g}_{\ell}^{(1)}\left(\frac{f_{\ell}(0)}{a}+f_{\ell}^{\prime}(0)\right)+o(1)
$$

as $a \downarrow 0$.

Proof We recall that $\tilde{V}_{V, n-1}(\Xi)$ is estimated from the data sampled on 1D section lattices. As a consequence, $\operatorname{dim} F_{\ell}$ is 0 or 1 . yields

Now plugging the result of Lemma 3 into Eq. 20

$$
\begin{aligned}
\mathbb{E} \tilde{V}_{V, n-1}(\Xi) & =\frac{1}{\alpha_{n 01} a} \sum_{\ell=0}^{v_{0}} \bar{g}_{\ell}^{(1)}\left(\mathbb{P}\left(F_{\ell} \subseteq \Xi^{c}\right)+o(a)\right) \\
& =\frac{1}{\alpha_{n 01} a} \sum_{\ell=0}^{v_{0}} \bar{g}_{\ell}^{(1)} f_{\ell}(a)+o(1) .
\end{aligned}
$$

The assertion of the theorem now follows from the series expansion Eq. 25.

Theorem 2 Let $\Xi$ be a Boolean model in $\mathbb{R}^{n}, n \geq 3$, with balls of random diameter $r$. If $r>\varepsilon>0$ a. s. and $\mathbb{E} r^{n}<\infty$, then

$$
\mathbb{E} \tilde{V}_{V, n-k}(\Xi)=\frac{(-1)^{k+1}}{\alpha_{n 0 k}} \sum_{\ell=0}^{v_{0}} \bar{g}_{\ell}^{(k)} \sum_{i=0}^{k} \frac{f_{\ell}^{(i)}(0)}{i ! a^{k-i}}+o(1)
$$

as $a \downarrow 0$ and for $k=1,2$.

Proof We make use of the fact that $\tilde{V}_{V, n-1}(\Xi)$ and $\tilde{V}_{V, n-2}(\Xi)$ are estimated from $1 \mathrm{D}$ and $2 \mathrm{D}$ section lattices, respectively, and thus $\operatorname{dim} F_{\ell} \leq 2$ meeting the assumptions of Lemma 4.

Then plugging the result of Lemma 4 into Eq. 20 and using Eq. 25 in the same way as in the proof of Theorem 1 yields Eq. 27. 


\section{BOOLEAN MODELS IN $\mathbb{R}^{3}$}

We specialise to the 3D case from now on. Let $\bar{V}, \bar{S}, \overline{\bar{b}}$ denote the expectations of the volume, the expectation of surface area, and the expectation of the mean breadth of the grain $\Xi_{1}$, i.e., $\mathbb{E} V_{3}\left(\Xi_{1}\right)=\bar{V}$, $2 \mathbb{E} V_{2}\left(\Xi_{1}\right)=\bar{S}$ and $\frac{1}{2} \mathbb{E} V_{1}\left(\Xi_{1}\right)=\overline{\bar{b}}$. Then the surface density $S_{V}=2 V_{V, 2}(\Xi)$, the density of the integral of the mean curvature $M_{V}=\pi V_{V, 1}(\Xi)$, and the density of the Euler number $\chi_{V}=V_{V, 0}(\Xi)$ of a Boolean model can be expressed in terms of $\lambda, \bar{V}, \bar{S}$, and $\overline{\bar{b}}$ by Miles' formulae

$$
\begin{aligned}
S_{V} & =\mathrm{e}^{-\lambda \bar{V}} \lambda \bar{S}, \\
M_{V} & =\mathrm{e}^{-\lambda \bar{V}}\left(2 \pi \lambda \overline{\bar{b}}-\frac{\pi^{2} \lambda^{2}}{32} \bar{S}^{2}\right), \\
\chi_{V} & =\mathrm{e}^{-\lambda \bar{V}}\left(\lambda-\frac{\lambda^{2}}{2} \overline{\bar{b}} \bar{S}+\frac{\pi \lambda^{3}}{384} \bar{S}^{3}\right),
\end{aligned}
$$

see Miles (1976) and Theorem 9.1.4 in Schneider and Weil (2008). On the other hand the expectations of the corresponding estimators according to Eqs. 26 and 27 have a structure similar to Miles' formulae,

$$
\begin{aligned}
\mathbb{E} \tilde{S}_{V} & =\mathrm{e}^{-\lambda \bar{V}} \lambda \bar{S}+o(1), \\
\mathbb{E} \tilde{M}_{V} & =\mathrm{e}^{-\lambda \bar{V}}\left(2 \pi \lambda \overline{\bar{b}}-0.354297 \lambda^{2} \bar{S}^{2}\right)+o(1), \\
\mathbb{E} \tilde{\chi}_{V} & \approx \mathrm{e}^{-\lambda \bar{V}}\left(\lambda^{2} \frac{c_{1}}{a} \bar{S}^{2}+\lambda+\lambda^{2} c_{2} \overline{\bar{b}} \bar{S}+\lambda^{3} c_{3} \bar{S}^{3}\right),
\end{aligned}
$$

where the constants $c_{1}, c_{2}$, and $c_{3}$ depend on the chosen pair of complementary adjacency systems $\left(\mathbb{F}, \mathbb{F}_{c}\right)$, see Table 4. Notice that the values for $c_{1}, c_{2}$, and $c_{3}$ correspond to those ones published in Ohser et al. (2003) for a Boolean model with balls of constant diameter.

In order to show that Eqs. 29 and 30 follow directly from Eqs. 26 and 27, respectively, we introduce the volume $v_{\ell}$, the surface area $s_{\ell}$, and the mean breadth $b_{\ell}$ of the set $\frac{1}{a} F_{\ell}$. Using these notations, the function $f_{\ell}$ can be rewritten as

$$
f_{\ell}(a)=\exp \left\{-\lambda\left(\bar{V}+\frac{1}{2} \bar{S} b_{\ell} a+\frac{1}{2} \overline{\bar{b}} s_{\ell} a^{2}+v_{\ell} a^{3}\right)\right\} .
$$

The first derivatives of $f_{\ell}$ at $a=0$ are

$$
\begin{aligned}
f_{\ell}(0) & =\mathrm{e}^{-\lambda \bar{V}} \\
f_{\ell}^{\prime}(0) & =\mathrm{e}^{-\lambda \bar{V}}\left(-\lambda \frac{1}{2} \bar{S} b_{\ell}\right) \\
f_{\ell}^{\prime \prime}(0) & =\mathrm{e}^{-\lambda \bar{V}}\left(-\lambda \overline{\bar{b}} s_{\ell}+\lambda^{2}\left(\frac{1}{2} \bar{S} b_{\ell}\right)^{2}\right), \\
f_{\ell}^{\prime \prime \prime}(0) & =\mathrm{e}^{-\lambda \bar{V}}\left(-\lambda 6 v_{\ell}+\lambda^{2} \frac{3}{2} \overline{\bar{b}} s_{\ell} \bar{S} b_{\ell}-\lambda^{3}\left(\frac{1}{2} \bar{S} b_{\ell}\right)^{3}\right) .
\end{aligned}
$$

For $k=1$, it can be seen from the values in the Tables 2 and 3 that

$$
\sum_{\ell=0}^{21} \bar{g}_{\ell}^{(1)}=0, \quad \sum_{\ell=0}^{21} \bar{g}_{\ell}^{(1)} b_{\ell}=-\frac{1}{2}
$$

which yields Eq. 29. For $k=2$ it follows that

$$
\begin{gathered}
\sum_{\ell=0}^{21} \bar{g}_{\ell}^{(2)}=0, \quad \sum_{\ell=0}^{21} \bar{g}_{\ell}^{(2)} b_{\ell}=0, \\
\sum_{\ell=0}^{21} \bar{g}_{\ell}^{(2)} b_{\ell}^{2}=0.451105, \quad \sum_{\ell=0}^{21} \bar{g}_{\ell}^{(2)} s_{\ell}=2,
\end{gathered}
$$

and from Eq. 27 one obtains Eq. 30.

Finally, consider the case $k=3$. Independent of the choice of a pair $\left(\mathbb{F}, \mathbb{F}_{c}\right)$ of complementary adjacency systems, it can be seen that

$$
\begin{array}{ll}
\sum_{\ell=0}^{21} \bar{g}_{\ell}^{(3)}=0, & \sum_{\ell=0}^{21} \bar{g}_{\ell}^{(3)} b_{\ell}=0, \\
\sum_{\ell=0}^{21} \bar{g}_{\ell}^{(3)} s_{\ell}=0, & \sum_{\ell=0}^{21} \bar{g}_{\ell}^{(3)} v_{\ell}=-1,
\end{array}
$$

which yields Eq. 31 where the coefficients $c_{1}, c_{2}, c_{3}$ are computed via

$$
c_{1}=\frac{1}{8} \sum_{\ell=0}^{21} \bar{g}_{\ell}^{(3)} b_{\ell}^{2}, \quad c_{2}=\frac{1}{4} \sum_{\ell=0}^{21} \bar{g}_{\ell}^{(3)} b_{\ell} s_{\ell}
$$

$$
c_{3}=\frac{1}{48} \sum_{\ell=0}^{21} \bar{g}_{\ell}^{(3)} b_{\ell}^{3}
$$

Table 3. The mean width $b_{\ell}$, the surface area $s_{\ell}$ and the volume $v_{\ell}$ for the convex hulls of the representatives $\eta_{\ell}$ of the 22 congruence classes. The constant $c$ is given by $c=\frac{1}{\pi} \arctan \sqrt{2}$. 


\begin{tabular}{|c|c|c|c|c|}
\hline$\ell$ & $\eta_{\ell}$ & $b_{\ell}$ & $s_{\ell}$ & $v_{\ell}$ \\
\hline 0 & 舟 & 0 & 0 & 0 \\
\hline 1 & $\xi_{1}$ वृी & 0 & 0 & 0 \\
\hline 2 & वीग्युग & $\frac{1}{2}$ & 0 & 0 \\
\hline 3 & $\xi_{9}$ वृ़ी & $\frac{1}{\sqrt{2}}$ & 0 & 0 \\
\hline 4 & $\xi_{129}$ & $\frac{\sqrt{3}}{2}$ & 0 & 0 \\
\hline 5 & $\xi_{11}$ & $\frac{1}{2}+\frac{1}{2 \sqrt{2}}$ & 1 & 0 \\
\hline 6 & $\xi_{131}$ & $\frac{1+\sqrt{3}}{4}+\frac{1}{2 \sqrt{2}}$ & $\sqrt{2}$ & 0 \\
\hline 7 & $\xi_{41}$ & $\frac{3}{2 \sqrt{2}}^{-12}$ & $\sqrt{3}$ & 0 \\
\hline 8 & $\xi_{15}$ & 1 & 2 & 0 \\
\hline 9 & $\xi_{43}$ & $\frac{3}{8}+\frac{3(1-c)}{2 \sqrt{2}}$ & $\frac{3+\sqrt{3}}{2}$ & $\frac{1}{6}$ \\
\hline 10 & $\xi_{139}$ & $\frac{1}{2}+\frac{1}{2 \sqrt{2}}+\frac{1}{2 \sqrt{3}}$ & $1+\sqrt{2}$ & $\frac{1}{6}$ \\
\hline 11 & $\xi_{153}$ & $\frac{1}{2}+\frac{1}{\sqrt{2}}$ & $2 \sqrt{2}$ & 0 \\
\hline 12 & $\xi_{105}$ & $3 \sqrt{2} c$ & $2 \sqrt{3}$ & $\frac{1}{3}$ \\
\hline 13 & $\xi_{99}$ & $\frac{3}{8}+\frac{1+3 c}{2 \sqrt{2}}+\frac{1}{4 \sqrt{3}}$ & $\frac{1}{2}+\sqrt{2}+\frac{\sqrt{3}}{2}$ & $\frac{1}{6}$ \\
\hline 14 & $\xi_{214}$ & $\frac{3}{8}+\frac{9 c}{2 \sqrt{2}}$ & $\frac{3(1+\sqrt{3})}{2}$ & $\frac{1}{2}$ \\
\hline 15 & $\xi_{124}$ & $\frac{5}{8}+\frac{1+3 c}{2 \sqrt{2}}$ & $\frac{3+\sqrt{3}}{2}+\sqrt{2}$ & $\frac{1}{3}$ \\
\hline 16 & $\xi_{248}$ & $\frac{3}{4}+\frac{1}{2 \sqrt{2}}+\frac{1}{4 \sqrt{3}}$ & $2+\sqrt{2}$ & $\frac{1}{3}$ \\
\hline 17 & $\xi_{126}$ & $\frac{3}{4}+\frac{3 c}{\sqrt{2}}$ & $3+\sqrt{3}$ & $\frac{2}{3}$ \\
\hline 18 & $\xi_{246}$ & $\frac{3}{4}+\frac{3 c}{\sqrt{2}}$ & $3+\sqrt{3}$ & $\frac{2}{3}$ \\
\hline 19 & $\xi_{252}$ & $1+\frac{1}{2 \sqrt{2}}$ & $3+\sqrt{2}$ & $\frac{1}{2}$ \\
\hline 20 & $\xi_{254}$ & $\frac{9}{8}+\frac{3 c}{2 \sqrt{2}}$ & $\frac{9}{2}+\frac{\sqrt{3}}{2}$ & $\frac{5}{6}$ \\
\hline 21 & $\xi_{255}$ [ी] & $\frac{3}{2}$ & 6 & 1 \\
\hline
\end{tabular}

Table 4. The values of the constants $c_{1}, c_{2}, c_{3}$ for the continuous case, cf. Eq. 28, and the four considered pairs of complementary adjacency systems.

\begin{tabular}{cllc}
\hline & $10^{3} c_{1}$ & \multicolumn{1}{c}{$c_{2}$} & $10^{3} c_{3}$ \\
\hline continuous & 0 & -0.5 & $8.181 \ldots$ \\
$\left(\mathbb{F}_{26}, \mathbb{F}_{6}\right)$ & 0 & -0.75 & 15.625 \\
$\left(\mathbb{F}_{14.1}, \mathbb{F}_{14.1}\right)$ & $-1.248 \ldots$ & $-0.105 \ldots$ & $12.770 \ldots$ \\
$\left(\mathbb{F}_{14.2}, \mathbb{F}_{14.2}\right)$ & $-3.294 \ldots$ & $-0.110 \ldots$ & $13.918 \ldots$ \\
$\left(\mathbb{F}_{6}, \mathbb{F}_{26}\right)$ & $-0.409 \ldots$ & $-0.547 \ldots$ & $-9.449 \ldots$ \\
\hline
\end{tabular}

In order to assess the asymptotic behaviour for $a \downarrow 0$ of the estimators $\hat{S}_{V}, \hat{M}_{V}$, and $\hat{\chi}_{V}$, Eqs. 29, 30, and 31 are compared with Miles' formulae. It can bee seen that $\hat{S}_{V}$ is asymptotically unbiased for $a \downarrow 0$ (multigrid convergent). This is related to the fact that the estimators of the Euler numbers of 1D Boolean models are asymptotically unbiased, see Serra (1982).

For Boolean models with balls of random diameters it can be shown that the asymptotic bias of $\hat{M}_{V}$ is

$$
\lim _{a \downarrow 0} \mathbb{E} \hat{M}_{V}-M_{V}=-0.045872 \lambda^{2} \bar{S}^{2} \mathrm{e}^{-\lambda \bar{V}}
$$

This corresponds to a result in Serra (1982), where the estimation of the Euler number of a planar Boolean model was shown to be asymptotically biased. In order to assess the asymptotic bias of $\hat{\chi}_{V}$, the constants $c_{1}, c_{2}, c_{3}$ have to be compared with the respective ones in Eq. 28. Obviously, $\hat{\chi}_{V}$ is always biased. More precisely, the asymptotic bias is even infinite for the pairs $\left(\mathbb{F}_{6}, \mathbb{F}_{26}\right),\left(\mathbb{F}_{14.1}, \mathbb{F}_{14.1}\right)$ and $\left(\mathbb{F}_{14.2}, \mathbb{F}_{14.2}\right)$.

For $\left(\mathbb{F}_{26}, \mathbb{F}_{6}\right)$ the coefficient $c_{1}$ in Eq. 31 vanishes and thus the difference of the right-hand sides of Eqs. 28 and 31 is finite. However, the error in Eq. 31 can not be estimated and thus the asymptotic behaviour of $\hat{\chi}_{V}$ is unknown for $\left(\mathbb{F}_{26}, \mathbb{F}_{6}\right)$.

\section{DISCUSSION}

The probably most intuitive method for measuring the surface area is based on rendering data, see, e.g., Lindblad and Nyström (2002), where the areas of the surface patches serve as weights for computing the surface area from local knowledge, i.e., from the numbers of pixel configurations. However, this type of estimator is not multigrid convergent. There are alternative methods also based on an explicit approximation of the boundary without further assumptions but ensuring multigrid convergence, see Klette and Rosenfeld (2004) for an overview. However, these approximations are expensive since they are not local.

As pointed out in Section 4.2, the surface area can be measured directly from the binary volume image without need to approximate the surface. We are starting from the local knowledge represented by the numbers of $2 \times 2 \times 2$ pixel configurations on cubic primitive lattices and ask for the best choice of surface weights. The weights suggested by Lindblad (2005) minimise the estimation variance of the surface area of a plane with random normal direction uniformly distributed on the unit sphere. This idea goes back to Mullikin and Verbeek (1993), see also the discussion in Windreich et al. (2003). A comprehensive treatment of the subject of surface estimation is due to Ziegel and Kiderlen (2009). Their weights minimise the asymptotic worst case error for surface area estimation for decreasing lattice distances (multigrid convergence). This approach is based on a general asymptotic result proved in Kiderlen and Rataj (2006). It also allows a comparison of the various methods for surface estimation. As shown in Ziegel and Kiderlen (2009), the maximum asymptotic relative error for general sets $X$ is $12.8 \%$ for the weights suggested in Lindblad and Nyström (2002), $7.3 \%$ for the weights given in Lindblad (2005) and Schladitz 
et al. (2006) and $4.0 \%$ for the best choice in Ziegel and Kiderlen (2009).

The approach based on the Crofton formulae has a number of advantages. First, the method of surface area estimation can simply be extended to arbitrary dimensions and to most of the other intrinsic volumes, in particular the integral of the mean curvature which measures e.g. the length of fibres in non-wovens or the total length of edges of open foams. Furthermore, this approach works for arbitrary homogeneous lattices so that we are no longer restricted to cubic primitive lattices, see Chapter 4 in Ohser and Mücklich (2000) and Lang et al. (2001). This is an important fact since many imaging techniques like nano-tomography by electron microscopy combined with focused ion beam slicing produce images on non-cubic lattices.

The diverging bias of the Euler number estimators of Boolean models is due to the huge number of tiny 'tunnels' and single background pixels between justtouching grains in discretisations. These tunnels and single pixels can be removed by smoothing the surface, e.g., with a morphological closure before determining the Euler number. On the other hand, when assuming alternative (and even more realistic) sampling models, e.g., suggested in Hall and Molchanov (1999), the bias in $\hat{\chi}_{V}$ is probably much smaller than for the $\mathbb{L}^{n}$ sampling considered in the present paper.

\section{ACKNOWLEDGEMENTS}

The authors very much appreciate fruitful comments by an unknown referee and Markus Kiderlen whose suggestions improved the paper considerably. Katja Schladitz was supported by the Rheinland-Pfalz cluster of excellence "Dependable Adaptive Systems and Mathematical Modelling" (http://www.dasmod.de/). The research of Joachim Ohser was supported by the $\mathrm{FH}^{3}$-programme of the German Federal Ministry of Education and Research under project grant 1711B06.

\section{REFERENCES}

Blasquez I, Poiraudeau JF (2003). Efficient processing of Minkowski functionals on a $3 \mathrm{~d}$ binary image using binary decision diagrams. In: Journal of WSCG, vol. 11 (1). WSCG, Plzen, Czech Republic: UNION AgencyScience Press.

Guderlei R, Klenk S, Mayer J, Schmidt V, Spodarev E (2007). Algorithms for the computation of Minkowski functionals of deterministic and random polyconvex sets. Image Vision Comput 25:464-74.

Hadwiger H (1957). Vorlesungen über Inhalt, Oberfläche und Isoperimetrie. Berlin: Springer-Verlag.
Hall P, Molchanov IS (1999). Corrections for systematic boundary effects in pixel-based area counts. Pattern Recogn 32:1519-28.

Jernot J, Jouannot-Chesney P, Lantuejoul C (2004). Local contributions to the Euler-Poincaré characteristic of a set. J Microsc 215:40-9.

Kiderlen M, Rataj J (2006). On infinitesimal increase of volumes of morphological transforms. Mathematica 53:103-27.

Klenk S, Schmidt V, Spodarev E (2006). A new algorithmic approach to the computation of Minkiwski functionals of germ-grain models. Comp Geom Th Appl 34:12748.

Klette R, Rosenfeld A (2004). Digital Geometry. Amsterdam: Morgan \& Kaufman Publ.

Lachaud JO, Montanvert A (2000). Continuous analogs of digital boundaries: A topological approach to isosurfaces. Graph Models 62:129-64.

Lang C, Ohser J, Hilfer R (2001). On the analysis of spatial binary images. J Microsc 203:303-13.

Lindblad J (2005). Surface area estimation of digitized $3 \mathrm{D}$ objects using weighted local computations. Image Vision Comput 23:111-22.

Lindblad J, Nyström I (2002). Surface area estimation of digitized 3D objects using local computations. In: 10th International Conference on Discrete Geometry for Computer Imagery, vol. 2301 of LNCS. DGCI, Bordeaux, France, Berlin, Heidelberg, New York: Springer.

Matheron G (1975). Random Sets and Integral Geometry. New York, London: John Wiley \& Sons.

Miles RE (1976). Estimating aggregate and overall characteristics from thick selections by transmission microscopy. J Microsc 107:227-33.

Molchanov IS (1997). Statistics of the Boolean Model for Practitioners and Mathematicans. New York: J Wiley \& Sons.

Mrkvička T, Rataj J (2008). On the estimation of intrinsic volume densities of stationary random closed sets. Stoch Proc Appl 118:213-31.

Mullikin J, Verbeek P (1993). Surface estimation of digital planes. Bioimaging 1:6-16.

Nagel W, Ohser J, Pischang K (2000). An integralgeometric approach for the Euler-Poincaré characteristic of spatial images. J Microsc 198:54-62.

Ohser J, Mücklich F (2000). Statistical Analysis of Microstructures in Materials Science. Chichester, New York: J Wiley \& Sons. 
Ohser J, Nagel W, Schladitz K (2002). The Euler number of discretized sets - on the choice of adjacency in homogeneous lattices. In: Mecke KR, Stoyan D, eds., Morphology of Condensed Matter, Lecture Notes in Physics. Berlin: Springer.

Ohser J, Nagel W, Schladitz K (2003). The Euler number of discretised sets - surprising results in three dimensions. Image Anal Stereol 22:11-9.

Rataj J, Zähle M (2003). Normal cycles of Lipschitz manifolds by approximation with parallel sets. Differ Geom Appl 19:113-26.

Schladitz K, Ohser J, Nagel W (2006). Measurement of intrinsic volumes of sets observed on lattices. In: Kuba A, Nyul LG, Palagyi K, eds., 13th Int Conf Discrete Geom Computer Imagery, LNCS. DGCI, Szeged, Hungary, Berlin, Heidelberg, New York: Springer.

Schmidt V, Spodarev E (2005). Joint estimators for the specific intrinsic volumes of stationary random sets. Stoch Proc Appl 115:959-81.
Schneider R (1993). Convex Bodies: The Brunn-Minkowski Theory. Cambridge: Encyclopedia of Mathematics and Its Application Vol. 44, Cambridge University Press.

Schneider R, Weil W (2008). Stochastic and Integral Geometry. Probability and Its Application. Heidelberg: Springer.

Serra J (1982). Image Analysis and Mathematical Morphology, Vol. 1. London: Academic Press.

Wills JM (1970). Zum Verhältnis von Volumen zu Oberfläche bei konvexen Körpern. Arch Math 21:55760 .

Windreich G, Kiryati N, Lohmann G (2003). Surface area estimation in practice. In: Nyström I, di Baja GS, Svensson S, eds., 11th Int Conf Discrete Geom Computer Imagery, vol. 2886 of LNCS. DGCI, Naples, Italy, Berlin, Heidelberg, New York: Springer.

Ziegel J, Kiderlen M (2009). Estimation of the surface area and surface area measure of three-dimensional sets from digitizations. Image Vision Comput, in press. 